\title{
PINK1 Regulates Dopamine and Lipids at Mitochondria to Maintain Synapses and Neuronal Function
}

Christine Bus1*, Sven Geisler ${ }^{*}$, Marita Feldkaemper ${ }^{2}$, Hector Flores-Romero ${ }^{3}$, Anna Schaedler ${ }^{1}$, Katharina Zittlau ${ }^{4}$, Maria Zarani ${ }^{1}$, Betül Uysal ${ }^{5}$, Nicolas Casadei ${ }^{6}$, Petra Fallier-Becker $^{7}$, Lisa Schwarz ${ }^{1}$, Jos F. Brouwers ${ }^{8}$, Henner Koch ${ }^{5}$, Aslihan UgunKlusek $^{9}$, Klaudia Maruszczak ${ }^{3}$, Daniela M. Vogt Weisenhorn ${ }^{10}$, Wolfgang Wurst $^{10,11,12}$, Benjamin Schmidt ${ }^{1}$, Gerard Martens ${ }^{13}$, Britta Brügger ${ }^{14}$, Doron Rapaport ${ }^{3}$, Ana Garcia ${ }^{3}$, Boris Macek ${ }^{4}$, Rejko Krüger ${ }^{15}$, Thomas Gasser ${ }^{1}$, Philipp Kahle $^{1}$, Julia C. Fitzgerald ${ }^{1}$

*Shared authorship

1 Department of Neurodegenerative Diseases, Centre of Neurology and Hertie Institute for Clinical Brain Research, University of Tübingen, Tübingen, Germany; 2 Institute for Ophthalmic Research, Centre for Ophthalmology, University of Tübingen, Tübingen, Germany; 3 Interfaculty Institute of Biochemistry, University of Tübingen, Tübingen, Germany; 4 Proteome Centre Tübingen, Interfaculty Institute for Cell Biology, University of Tübingen, Tübingen, Germany; 5 Department of Department Neurology and Epileptology, Centre of Neurology and Hertie Institute for Clinical Brain Research, University of Tübingen, Tübingen, Germany; . BZH Heidelberg University Biochemistry Center, Heidelberg, Germany; 6 Institute of Medical Genetics and Applied Genomics, University of Tübingen, Tübingen, Germany; 7 Institute of Pathology and Neuropathology, University of Tübingen, Tübingen, Germany; 8 Dept. Biochemistry \& Cell Biology, Lipidomics Facility, Faculty of Veterinary Medicine, Utrecht University, Yalelaan 2, 3584 CM Utrecht, The Netherlands; 9 School of Science and Technology, Nottingham Trent University, Nottingham, UK; 10 Helmholtz Zentrum München, German Research Center for Environmental Health, Institute of Developmental Genetics, München-Neuherberg, Germany; 11 Technische Universität München-Weihenstephan, Chair of Developmental Genetics, Munich, Germany; 12 German Center for Neurodegenerative Diseases (DZNE), Site Munich, Munich, Germany; 13 Department of Molecular Animal Physiology, Nijmegen Center for Molecular Life Sciences (NCMLS), Radboud University, Nijmegen, The Netherlands; 14 BZH Heidelberg University Biochemistry Center, Heidelberg, Germany; 15 Luxembourg Centre for Systems Biomedicine (LCSB), University of Luxembourg, Esch-sur-Alzette, Luxembourg and Parkinson Research Clinic, Centre Hospitalier de Luxembourg $(\mathrm{CHL})$, Luxembourg Luxembourg Institute of Health $(\mathrm{LIH}$, Luxembourg);

Corresponding author: Julia C. Fitzgerald, Hertie Institute for Clinical Brain Research, Otfried Müller Strasse 27, 72076, Tübingen, Germany. Email: julia.fitzgerald@uni-tuebingen.de, Tel: +49 7071298197

Abstract word count: 266 


\section{Abstract}

Mitochondrial dysfunction contributes to the pathogenesis of Parkinson's disease but it is not clear why inherent mitochondrial defects lead specifically to the death of dopaminergic neurons of the mid brain. PINK1 is mitochondrial kinase and PINK1 mutations cause early onset Parkinson's disease.

We found that in neuronal progenitors, PINK1 regulates mitochondrial morphology, mitochondrial contact to the endoplasmic reticulum (ER) and the phosphorylation of Miro1. A compensatory metabolic shift towards lipid synthesis provides mitochondria with the components needed for membrane renewal and oxidative phosphorylation, maintaining the mitochondrial network once mature.

Cholesterol is increased by loss of PINK1, promoting overall membrane rigidity. This alters the distribution of phosphorylated DAT at synapses and impairs dopamine uptake. PINK1 is required for the phosphorylation of tyrosine hydroxylase at Ser19, dopamine and calcium homeostasis and dopaminergic pacemaking.

We suggest a novel mechanism for PINK1 pathogenicity in Parkinson's disease in addition to but not exclusive of mitophagy. We also provide a basis for potential therapeutics by showing that low doses of the cholesterol depleting drug Bcyclodextrin reverse PINK1-specific phenotypes.

\section{Introduction}

PINK1 mutations are the second most frequent cause of early-onset PD and PINK1 variants have been found in sporadic PD but also in healthy controls ${ }^{1,2}$. PINK1 PD has an early occurrence of L-DOPA-associated dyskinesia, slow disease progression and absence of cognitive impairment ${ }^{3,4}$ and affective and psychotic symptoms are frequently part of the clinical presentation ${ }^{5}$. 
To unravel the molecular function of PINK1, Knockouts (KOs) of the PINK1 gene in mice, drosophila, zebrafish and cancer cells have been generated indicating that PINK1 deficiency causes mitochondrial dysfunction, altered mitochondrial morphology, disturbed mitochondrial quality control and iron and calcium toxicity ${ }^{6-15}$. In PINK1 in vivo models, neurodegeneration has only been observed in zebrafish ${ }^{16,17}$ with dopamine release and synaptic plasticity being affected in the striatum of mice ${ }^{18}$.

PINK1 acts together with another PD gene product, the E3-ubiquitin ligase Parkin ${ }^{19}$ 20 to regulate mitophagy ${ }^{21-23}$. Upon loss of mitochondrial membrane potential or accumulation of misfolded proteins, PINK1 stabilizes on the outer mitochondrial membrane ${ }^{23-25}$. PINK1 then phosphorylates ubiquitin at Ser65 to activate Parkin activity $^{26-28}$. Parkin ubiquitinylates mitochondrial outer membrane proteins such as Mitofusins $^{29}$ and Miro ${ }^{30}$ which is also targeted for phosphorylation ${ }^{31}$. The buildup of ubiquitin chains on the outer mitochondrial membrane acts as a signal for the recruitment of autophagy receptors ${ }^{21,32,33}$ important for mitochondrial clustering ${ }^{34,35}$. Some in vivo studies have shown that PINK1 is not required for basal mitophagy in neurons $^{36,37}$ or in human platelets ${ }^{38}$ but the topic is controversial (for an extensive review see $^{39,40}$ ) and therefore much more work is needed.

The pathogenic action of PINK1 mutations could be cell type specific. PINK1 is highly expressed in the brain but it is also expressed throughout the rest of the body and has been associated with disease mechanisms in several tissues ${ }^{41,42}$ including the progression of some cancers ${ }^{14,43}$. PINK1 is reportedly expressed predominantly in neurons ${ }^{44}$ but has been found highly expressed in myelinating oligodendrocytes in the human cortex ${ }^{45}$. First studies in human PINK1 patient and stem cell-derived neurons described defective Parkin recruitment to mitochondria and increased mitochondrial biogenesis ${ }^{46}$. 
Although autophagosomes are generated at ER-contact sites ${ }^{47}$ PINK1 may play important roles in addition to mitophagy at mitochondrial-ER contact sites and in ER stress $^{48-50}$. Proteins such as Miro1, VDAC and mitofusins have been strongly linked to $\mathrm{ER}$ and PINK1 function relevant to $\mathrm{PD}^{21,51-54}$. Mitochondrial-ER contact sites are regulatory hubs for calcium, iron, lipids and dopamine. PINK1 loss of function causes calcium defects in neurons ${ }^{9,17,55}$ and iron toxicity ${ }^{6,56-58}$. A PINK1-specific RNAi screen identified lipid regulation as a key factor in PINK1 mechanism of action ${ }^{59}$, The lipid cardiolipin externalizes on the mitochondrial outer membrane as a signal for mitophagy $^{60}$ and promotes the transfer of electrons to complex I to protect against PINK1 loss of function ${ }^{61}$. PINK1 also regulates the enzyme cardiolipin synthase ${ }^{62}$. Dopamine synthesis and degradation is primarily regulated at the mitochondrial outer membrane and ER because of the localization of monoamine oxidase (MAO) and tyrosine hydroxylase (TH), which require ubiquitination ${ }^{63,64}$ and phosphorylation (for a review see ${ }^{65}$ ) for their activation respectively.

Recently PINK1 has been shown to mediate STING-induced inflammation ${ }^{66}$ and mitophagy associated with innate immunity via mitochondrial release of damage factors $^{67}$. Furthermore, PINK1 KO mice are vulnerable to infection which induces PDlike symptoms ${ }^{68}$. Cholesterol, fatty acids and other modified lipids are well known to activate inflammatory pathway and therefore more work is needed to confirm PINK1s mechanisms relevant to PD.

We find that PINK1 plays an important regulatory role in fine tuning mitochondrial contacts, lipid synthesis, membrane fluidity, calcium homeostasis and dopamine metabolism targets including Miro1, $\mathrm{TH}$ and mitochondrial Aconitase at the mitochondria and set out a mechanism involving a metabolic shift towards cholesterol 
synthesis, impacting neurotransmitter uptake and dopamine metabolism, providing a link between PINK1-mitochondrial function and dopaminergic vulnerability in PD.

\section{Results}

\section{Absence of PINK1 in hDANs inhibits ionophore-induced mitophagy but does not affect mitochondrial morphology}

We generated iPSCs from a healthy female individual that we previously characterised $^{69}$ and then introduced a homozygous deletion of PINK1 using TALEN directed to Exon 1 (Figure 1A). 28-35 day old human mid-brain specific dopaminergic neurons (hDANs) were derived via neural precursor cells (NPCs) using a modified version of a differentiation protocol we previously described ${ }^{69}$. We selected two clonal lines that were correctly edited with no random integration where no PINK1 transcripts upstream or downstream of the gene edit could be detected (Figure S1A). The derived hDAN cultures varied in their neuronal subtype markers between independent differentiations. hDANs presented staining levels for mature neuronal markers (Figure S1B). The neuronal marker MAP2 was abundant but the percentage of hDAN cultures positive for the dopaminergic neuron marker; forkhead-box-protein A2 (FOXA2), Tyrosine Hydroxylase (TH) and Dopamine Transporter (DAT) differed (Figure 1B). The average percentage of $\mathrm{TH}$ positive neurons in all hDAN cultures was approximately $25 \%$ (Figure 1B). Similar to the immunocytochemical data, $\mathrm{TH}$ gene expression was increased different between hDAN genotypes.

PINK1 KO has no impact on autophagic flux in hDANs (Figure 1D), but markers of classical, CCCP-induced mitophagy such as ubiquitination of mitofusin and degradation of TOM70 were strongly reduced following a time course of $10 \mu \mathrm{M}$ CCCP treatment (Figure 1E). Steady state levels of Miro1 and OPA1 remain largely 
unchanged by PINK1 KO (Figure 1E). We employed the ionophore Valinomycin to promote mitophagy over a longer $24 \mathrm{~h}$ time course to be able to fix cells (with less variability) for electron microscopy (EM). EM images from independent differentiations concluded there to be no notable differences of any organelles including mitochondrial size, shape, or cristae in untreated PINK1 KO hDANs (Figure 1F). Following mitophagy induction with Valinomycin, mitochondria (green arrows) were scarce in control hDANs, whilst mitochondria remain visible in PINK1 KO hDANs receiving the same treatment (Figure $1 \mathrm{~F}$ ). The appearance of structures resembling mitochondrial derived vesicles (MDVs) appear to increase in PINK1 KO hDANs treated with the ionophore (red arrow). Inhibited mitophagy by PINK1 KO in combination with ionophores leads to increased PGC1a levels, mitochondrial biogenesis (measured by the ratio of nuclear encoded SDHA : mitochondrial encoded MTCO1 proteins) and mitochondrial renewal (measured by the ratio of oxidized : nonoxidised mitochondrially expressed MitoTimer protein on the mitochondrial outer membrane) compared to control hDANs receiving the same treatment (Figure 1G) suggestive of compensatory PINK1-independent mitophagy and/or mitochondrial renewal.

\section{PINK1 knockout promotes metabolic rewiring towards lipid synthesis in hDANs}

We next looked at whether PINK1 is important for mitochondrial function. Mitochondrial oxygen consumption and spare respiratory capacity are not significantly affected although PINK1 KO hDANs consume more oxygen when uncoupled and prefer glycolysis under minimal respiration conditions than their control hDANs (Figure 2A). PINK1 KO hDANs prefer not to use fatty acids for oxygen consumption (Figure 2A). These data suggest that PINK1 KO hDANs do not well metabolize acetyl CoA from glucose nor fatty acids. We asked whether complex I (CI) 
dysfunction may explain the shift and $\mathrm{Cl}$ dysfunction has previously been associated with PINK1-PD $61,70,71 . \mathrm{Cl}$ enzyme activity in hDANs is not significantly affected by PINK1 KO (Figure 2B). However, since we observed variable citrate synthase enzyme activity (Figure 2C) which is used for normalization of mitochondrial mass for $\mathrm{Cl}$ activity assays, a possible role of complex I in the PINK1 mechanism cannot be ruled out. We also found no measurable differences in the amount of active $\mathrm{Cl}$ enzyme pulled down from untreated control or PINK1 KO hDANs (Figure S1C) and active $\mathrm{Cl}$ (which is depleted in control hDANs following $24 \mathrm{~h}$ valinomycin treatment) is unaffected by valinomycin in the absence of PINK1 (Figure S1C).

The mitochondrial membrane potential $\left(\Delta \Psi_{\mathrm{m}}\right)$ of PINK1 KO hDANs is reduced by approximately $15 \%$ in the basal state and is less responsive to mitochondrial toxins in live cell imaging (Figure 2D). The phenotype was confirmed using live cell flow cytometry methods (Figure S1D). PINK1 KO hDANs do not show any significant oxidative stress, with regards to mitochondrial reactive oxygen species (ROS) (Figure 2E). Additionally, cytosolic ROS (Figure S1E), lipid peroxidation (Figure S1F), antioxidant status and glutathione depletion (Figure S1G) are also unaffected by PINK1 KO.

A metabolic shift away from oxidative phosphorylation is accompanied by $\sim 50 \%$ inhibition of the iron-sulfur cluster, TCA enzyme Aconitase (Figure 2F), which is in line with previous work in vivo ${ }^{6}$ and suggestive of upregulated citrate synthase and increased shuttling of citrate out of the mitochondria. Downstream in the TCA cycle, a-ketoglutarate dehydrogenase enzyme activity $(\alpha \mathrm{KGDH})$ is unaffected by PINK1 KO (Figure $\mathrm{S} 1 \mathrm{H}$ ). Significantly increased $\mathrm{NAD}^{+}$(Figure $2 \mathrm{G}$ ) but not NADH (Figure S1I) reflects metabolic shifting as an adaptive response. 
We performed RNA sequencing in PINK1 KO hDANs in the basal state or following depolarization of the mitochondrial membrane with the ionophore Valinomycin to identify genes and pathways relevant to PINK1. The heatmap illustrates the most differentially regulated genes (log2 fold change) in both PINK1 KO hDAN clonal lines compared to their healthy control (Figure S2A). Pathway analysis comparing genotype revealed enrichment for genes involved in lipid synthesis and storage such as ELOV2/6 (long chain fatty acid (FA) synthesis), CYP27B1/CH25H (cholesterol regulation), FABP7 (FA binding and metabolism), COL1A2/COL4A4/COL4A2 (adipogenesis and fat storage), VCAM/LAMA1 (adhesion molecules involved in lipid metabolism and immune response) (Figure $2 \mathrm{H}$ ). Interestingly, ionophore treatment of control neurons induces predominant expression changes in genes involved in amino acid metabolism, gluconeogenesis, glycolysis, TCA and iron homeostasis (Figure $2 \mathrm{H})$.

Following on from these findings, we questioned whether lipid synthesis might be required for regeneration and biogenesis of mitochondrial membranes in the absence of PINK1 and asked whether specific lipids might be affected. We first measured cholesterol levels in whole cell preparations (Figure 2I) and then performed lipidomics on crude mitochondrial preparations (Figure $2 \mathrm{~J}$ ) and whole hDANs (Figure $2 \mathrm{~K}$ ). Cholesterol levels are increased by approximately $30 \%$ in PINK1 KO hDANs (Figure 2I), accompanied by a small increase in mitochondrial enriched samples which is not statistically significant (Figure S2B). Cholesterol and cardiolipins (CL) (Figure 2J) are also elevated in the crude mitochondrial preparations from PINK1 KO hDANs but the difference again is not significant. Lipidomics performed in whole hDANs showed both up and down regulation of lipid species in PINK1 KO lines (Figure S2C). Two way ANNOVA analysis of certain phosphatidylethanolamines (PE), 
phosphatidylcholines (PC), sphingomyelins (SM), CL and a ceramide b species where PINK1 KO hDANs have less than control, revealed significance but only for one of the two PINK1 KO clonal lines (Figure 2K, left panel). Upregulated lipid groups were significantly different to controls in both PINK1 KO clones. These lipids include: phosphatidyinositols (PI), PE, PS, PC, SM, CL and Bis(monoacylglycero)phosphates (BMP) (Figure 2K, right panel). Increased levels of phosphatidylethanolamines were significantly upregulated in statistical analysis of the individual lipid groups from whole hDAN analyses. Taken together, these data suggest a significant shift towards lipid abundance, which does not significantly alter the mitochondrial lipid pool. Cholesterol is most abundant at the plasma membrane and mitochondria only account for a small proportion of total cell cholesterol. Insulin-induced gene 1 protein (INSIG) and super conserved receptor expressed in brain (SREB) regulation at the ER may be relevant but requires much further work (especially the regulation of INSIG). Nevertheless, we could not detect any obvious differences in SREB colocalization with nuclei (Figure S2D). Total cholesterol levels were significantly increased in the striatum of four month old PINK1 KO mice (Figure S3C).

\section{PINK1 mechanisms switch during neuronal differentiation involving the phosphorylation of substrates at mitochondria and endoplasmic reticulum}

We hypothesized that PINK1 could have additional or overlapping roles in mitochondrial quality control acting via substrates at the mitochondrial outer membrane (MOM), Endoplasmic reticulum (ER) or mitochondrial associated membranes (MAM) that could explain the metabolic changes observed. First we measured the distances between mitochondria and ER using quantitative BRET signals and found that PINK1 KO significantly tightens the connections in NPCs which loosen in post-mitotic mature hDANs compared to controls (Figure 3A). 
Broader distance between mitochondria and ER reflects the glycolytic, lipid rich metabolic state observed in caloric excess and PINK1 KO hDANs. Furthermore, the ER of PINK1 KO hDANs fail to release normal amounts of calcium in response to thapsigargin treatment (Figure 3B). Tighter mitochondrial contacts in PINK1 KO NPCs is accompanied by significantly smaller mitochondria, suggestive of fragmentation (Figure 3C). Interestingly, PINK1 KO hDANs show no mitochondrial fragmentation (Figure 3C) nor any other changes in mitochondrial morphology compared to controls using live cell microscopy (Figure S2E).

Next we studied the phosphorylation status of Miro1, a known substrate of PINK1 which is important for mitochondrial-ER contact, mitochondrial morphology and mitochondrial movement. Phosphorylation of Miro1 is largely undetectable unless an ionophore is used to initiate PINK1 accumulation at the MOM. In control hDANs, phosphorylation events on Miro1 accumulate during CCCP treatment. Several phosphorylated Miro1 bands are absent in PINK1 KO lysates run on PhosTag gels (Figure 3D). PINK1 KO affects the phosphorylation of Miro1 more strongly in NPCs than in hDANs (Figure 3E). Grp75 and TOM70 may also be phosphorylated in the presence of PINK1 and this may again be more important in NPC intermediates (Figure S2F).

To explain PINK1 KO phenotypes in hDANs, we looked for putative PINK1 targets using quantitative phospho-proteomics on crude mitochondrial preparations. We normalized phospho-peptide abundance to the non-phospho proteome in the same samples and analyzed untreated hDANs and those treated with the cholesterol lowering drug Simvastatin to identify any putative substrates relevant to PINK1 regulation of lipid metabolism. We ranked phospho-protein hits based on those with the greatest log2 fold change and removed those that did not replicate in both PINK1 
$\mathrm{KO}$ clones (Figure S2G). To focus on interactions that may occur at the mitochondrial contact sites, we then listed phospho-proteins by the GO terms; mitochondria, ER, mitochondria and ER (Figure 3F). We identified GAP43 (Neuromodulin) which is a protein associated with calcium signaling and neuronal development and validated biochemically by PhosTag gels (Figure $\mathrm{S} 2 \mathrm{H}$ ). Of note, proteins related to iron and hormone regulation such as PGRMC1, an ER protein which is part of the ferrechelotase complex, was also validated (Figure $\mathrm{S} 2 \mathrm{H}$ ). Mitoferrin2, a protein involved in iron import into mitochondria is upregulated in PINK1 KO hDANs (Figure 3G). We monitored the mitochondrial uptake of labelled proteins and found no significant impairment in the absence of PINK1 (Figure S3D).

Finally, we identified that PINK1 could be important for the phosphorylation of tyrosine hydroxylase (TH) at serine 19 (Figure 3F), a protein filtered from phosphoproteomics data based on its GO terms (it localizes to both mitochondria and ER). We biochemically validated the event in PINK1 KO NPCs and hDANs. Missing and altered phospho-TH banding is observed in PINK1 KO hDANs on PhosTag gels (Figure 3G). PINK1-dependent phosphorylation of TH occurs in untreated hDANs and depolarization of the mitochondrial membrane with CCCP does not increase phosphorylation at the same band (Figure 3G), suggesting that PINK1 may not directly phosphorylate $\mathrm{TH}$ and membrane depolarization may be more important.

\section{PINK1 KO induced membrane rigidity alters the distribution and regulation of the dopamine transporter}

Upregulation of Rab3B expression was detected following RNA sequencing by qRTPCR (Figure S3A). Rab GTPases are known PINK1 targets ${ }^{72}$ and Rab8A is involved in recycling cholesterol to the plasma membrane ${ }^{73}$. We therefore questioned whether deregulation of cholesterol reported here could impact lipid membranes crucial for 
synaptic function in neurons. We used dynamic light scattering to measure the size and heterogeneity of membrane particles following mechanical homogenization of hDANs in the absence of detergents. We found PINK1 KO hDANs contain more disperse, larger particles compared to their healthy control (Figure 4A). Next we fractionated hDAN lysates by density and used marker proteins to identify the soluble fractions (8-14) and the cholesterol-rich floating fractions (3-7) marked by flotillin (Figure 4B). We assessed the distribution of several membrane-bound and cytosolic proteins in precipitated fractions from hDANs. Quantification of protein levels per fraction revealed that the lipid raft marker flotillin is distributed more heavily in the floating fractions indicative of increased cholesterol rich rafts in PINK1 KO hDANs (Figure 4C, upper panel). The distribution of ERK1/2, which is an abundant cytosolic protein is unaffected by PINK1 KO (Figure 4C, bottom panel) suggesting that membrane bound proteins may be particularly affected by changes in cholesterol. Since the dopamine transporter DAT is known to be negatively regulated by its phosphorylation in cholesterol rich lipid membrane rafts $^{74}$ reviewed $\mathrm{in}^{75}$, we then assessed the distribution of phosphorylated DAT and total DAT. PINK1 KO leads to more phosphorylated DAT in less soluble fractions (Figure 4D), which leads to increased DAT internalization. We treated hDANs with $1 \mathrm{mM} \beta$-cyclodextrin to deplete cholesterol, break up lipid rafts and push proteins into the soluble fractions as a technical control (Figure 4E). In collaboration with the authors of Jazaro et al. (submitted to NCB together with this manuscript) who had independently found that sub-lethal doses $\beta$-cyclodextrin reverse PINK1-specific phenotypes in patient derived models via unbiased compound screening, we then used nanomolar concentrations of $\beta$-cyclodextrin as a chronic treatment in the maturation media of hDANs and found that it was capable of reversing the reduced mitochondrial membrane potential 
previously observed (Figure 4F) also reduced utilization of exogenous fatty acids as an energy source (Figure $4 \mathrm{G}$ ).

\section{PINK1 knockout significantly affects dopamine uptake and neurotransmission}

We observed differences in DAT distribution and TH phosphorylation in PINK1 KO hDANs. We also observed altered expression of genes involved in dopamine metabolism and synaptic function. Of particular interest, upregulation of pyridoxine-5'phosphate oxidase (PNPO), which is important for the synthesis of amino acids and dopamine via AADC, and of $\mathrm{TH}$, which is important in dopamine synthesis, where observed (Figure S3B and Figure 1C). We therefore measured amine neurotransmitter metabolites in control and PINK1 KO hDANS. L-DOPA is routinely used in cell culture models to feed the system a substrate in order to measure dopamine (DA) and DA flux accurately using HPLC. The PINK1 KO hDANs have significantly reduced DOPAC and DA levels (Figure 5A). The ratio of DOPAC/DA was also reduced but not HVA/DA suggesting the importance of the pre-synapse (Figure 5B). We controlled for amount of dopaminergic neurons in the cell cultures by monitoring $\mathrm{TH}$ on mRNA and protein level in each experiment, which was not decreased in PINK1 KO hDANs (Figure 5C). DA levels inside hDANs and in the media could not be replenished by blocking degradation (Figure 5D). MAO A and B enzyme activity and MAO-A protein levels were not significantly affected by PINK1 $\mathrm{KO}$ (Figure 5E). However, since MAO levels are normally highly fluctuating, a possible functional role of the enzymes need to be further addressed.

Neurotransmitter uptake was significantly impaired in PINK1 KO hDANs (Figure 5F and Figure 5G) and this could not be rescued by inhibition of uptake in the vesicles (VMAT), DA synthesis via TH or DA degradation (COMT/MAO) (Figure 5H). We measured the amount of oxidized catecholamines and did not observe any 
differences between PINK1 KO hDANs and their control with or without L-DOPA treatment (Figure $5 \mathrm{H}$ ). Therefore, DA loss cannot be explained by oxidation but conversion to neuromelanin is still possible but difficult to detect in iPSC-derived 2D hDAN cultures.

Next we tested the electrophysiological properties of PINK1 KO hDANs using patch clamp recordings. Healthy hDANs showed consistent burst firing patterns, while PINK1 KO hDANs showed $50 \%$ spontaneous bursting or $50 \%$ no spontaneous action potentials (Figure 5I). PINK1 KO hDANs have a significantly elevated mean average resting membrane potential above $-50 \mathrm{mV}$ (Figure 5J). PINK1 KO hDANs generated action potentials when we artificially lowered their membrane potential to $-70 \mathrm{mV}$, the normal resting potential of healthy cells. In this state PINK1 KO hDANs were able to produce spontaneous bursting behavior similar to the healthy controls. However, these PINK1 KO hDANs have an increased amplitude of the bursting $(5.64 \pm 1.05$ control vs $15.26 \pm 12.26$ PINK1 KO and $14.39 \pm 1.63$ PINK1 KO) and often showed depolarization blocks within the bursting (Figure 5I, lower panel). PINK1 KO hDANs have an abnormal and hyper-excitable phenotype. Jazaro et al. (submitted together with this manuscript) found that abnormal firing patterns in PINK1 patient derived models can be rescued by low doses of $\beta$-cyclodextrin, suggesting that plasma membrane fluidity may be an important factor in PINK1 PD etiology.

\section{Discussion}

It is widely accepted that wild type PINK1 acts as a mitochondrial sensor, an initiator of stress responses and is important for mitochondrial quality control. We show here that PINK1 is not essential for the maintenance of mitochondrial networks or mitochondrial respiratory function in mature human neurons, which is in line with prior evidence that mitophagy can occur independently of PINK1 ${ }^{37,} 56, \quad 76$.PINK1 
orchestrates a network of fine tuning mechanisms such as PINK1-Parkin mediated mitophagy, inflammation and mitochondrial movement ${ }^{21,23,66,77}$. Here we show that PINK1 is also important for mitochondrial metabolism. Metabolic control within the mitochondrial matrix might be imposed by PINK1 via several mechanisms; firstly, via inner mitochondrial targets such as Aconitase, $\mathrm{Cl}$ or TRAP1 which have all been previously proposed ${ }^{6,70,78}$. PINK1 could indirectly regulate the mitochondrial import of iron or other molecules required as enzymatic co-factors. This is an interesting topic that requires additional work to go beyond protein import into mitochondria. PINK1 is known to regulate Miro1, which is a MOM protein crucial for calcium sensing, movement and mitophagy. It would be interesting to detangle the boundaries between mitophagy and mitochondrial-ER contact regulation since many PINK1-Parkin targets have functions spanning both processes.

Mitochondrial fragmentation and compensation occurring in developing neurons, which is overcome for the survival of the mature neurons supports the model that fission protects healthy mitochondrial domains from elimination when unchecked by the PINK1-Parkin pathway ${ }^{79}$, PINK1-independent mitophagy and alternative mitochondrial quality control pathways such as piecemeal mitophagy ${ }^{80}$ and mitochondria derived vesicles ${ }^{81}$. Here, the synthesis of lipids for renewal of the membranous mitochondrial network may be relevant.

We propose that neurons lacking PINK1 perform a citrate shunt catalyzing Acetyl CoA production and fatty acid synthesis. Mitochondrial-ER contacts in developing neurons could be favored to increase mitochondrial capacity by via B-oxidation ${ }^{82}$. Here oxidative damage is absent and the increased NAD+ pool further underscores the relevance of an adaptive response in early differentiation. 
In mature neurons distant mitochondrial-ER contacts reflect a high fed state and hyperlipidemia ${ }^{82}$. In an extensive metabolomics study of PD patients, changes in long chain acyl carnitines and their role in $\beta$-oxidation were highlighted as a robust PD biomarker ${ }^{83}$ and lipid regulating proteins were the most significant modifiers of PINK-mediated mitophagy in another unbiased screen ${ }^{59}$.

Cholesterol is one of the major components of lipid rafts which are crucial at the synapse and post synapse. Lipid rafts control the movement and modification of receptors and transporters but are also involved in endocytosis, exocytosis and vesicular transport. Previously Rab8A has been identified as a target of PINK1 ${ }^{72}$ which is involved in membrane transport but also in cholesterol recycling ${ }^{73}$. This pathway may be crucial for PINK1-specific mechanisms outside of mitochondria and the link to the synapse.

Altered dopamine uptake and calcium mismanagement contribute to the abnormal firing patterns and very high resting membrane potentials in PINK1 KO hDANs. We cannot rule out that low dopamine metabolites are caused by the additional impact of catechol estrogens and other lipid hormones on neurotransmitter synthesis ${ }^{84,85}$ or dopamine synthesis via AADC which requires PLP, a product of the vitamin B6 salvage pathway which is significantly down regulated in PINK1 KO hDANs at the enzyme PNPO. PLP is abundant in the base medias used for neuronal differentiations and therefore a different approach is required. PLP is crucial for amino acid, nucleotide and neurotransmitter synthesis ${ }^{86}$. Enhancing nucleotide metabolism was previously found to protect against PINK1-PD ${ }^{87}$ and therefore could be an entry point for therapeutics. PINK1 specific modification of $\mathrm{TH}$ at the mitochondrial membrane or at MAMs provides addition burden to dopaminergic neurons and the PD gene product $\alpha$-synuclein has previously been implicated in $\mathrm{TH}$ 
regulation at mitochondrial-ER contact $\operatorname{sites}^{88}$. Parkin is known to suppress $\mathrm{MAO}^{89}$, a mitochondrial anchored enzyme responsible for dopamine degradation and mitochondrial DNA damage ${ }^{90}$ and which is implicated in the pathogenesis of $\mathrm{PD}^{91}$. Our data underscores the relevance of dopamine regulation at the mitochondria in human neurons.

Here, the solubilizing agent ß-cyclodextrin (BCD) depleted cholesterol and therefore the lipid rafts (according to ${ }^{92}$ ) and was protective at low doses. Jarazo and colleagues (in the accompanying paper in this submission) found that BCD rescues mitochondrial and neuronal phenotypes in PINK1 patient-derived models. The mechanism of BCD action could be relevant for other forms of PD or neurodegeneration. Indeed, mild membrane cholesterol reduction also impacts the cleavage of APP mediated by APP trafficking and partitioning into lipid rafts ${ }^{93}$. Although Simvastatin use for brain related diseases is complicated by the dissociation of cholesterol metabolism in the brain (reviewed in ${ }^{94}$ ), data taken from the Norwegian medical database shows Simvastatin is associated with decreased risk of Parkinson's disease ${ }^{95}$. Further mechanistic work will determine whether BCD could be beneficial in other human neuronal models of Parkinson's disease.

\section{Materials and Methods}




\section{Ethics statement}

Human samples were obtained with consent and prior ethical approval at The University of Tübingen and the Hertie Institute for Clinical Brain Research Biobank.

Animal experiments were carried out in strict accordance with the recommendations in the Guide for the Care and Use of Laboratory Animals of the European Union and of the Federal Republic of Germany (TierSchG). The protocol was approved by the Institutional Animal Care and Use Committee (Ausschuss für Tierversuche und Versuchstierhaltung, ATV) of the Helmholtz Zentrum München. All efforts were made to minimize suffering.

\section{Generation of iPSC and PINK1 Knockout}

Human iPSCs were cultured in self-made E8 media on Vitronectin (VTN-N, Gibco) coated cell culture dishes. iPSCs from a healthy individual that were previously characterized ${ }^{96}$ were transfected with a TALEN and a homologous construct for PINK1 exon1 using an Amaxa Nucleofector II with the Stem cell Nucleofection Kit (both from Lonza). The transfected iPSCs were plated on VTN-N -coated $10 \mathrm{~cm}$ dishes in E8 medium containing $10 \mu \mathrm{M}$ ROCK inhibitor Y27632. Homologous recombined iPSC colonies were selected with $250 \mu \mathrm{g} / \mathrm{ml} \mathrm{G418} \mathrm{(Biochrom)} \mathrm{or}$ $10 \mu \mathrm{gml}-1$ Blasticidin (InvivoGen) in the second round of TALEN transfection and replated in 12-well plates. Resistant iPSC colonies were characterized by sequencing, qRT-PCR and Western blot to confirm successful homozygous gene knockout. TALENs were designed with the online tool TAL Effector Nucleotide Targeter 2.0 (Cornell University) and generated using a cloning protocol adapted from ${ }^{97}$. The following RVD sequences were used for the TALEN monomers: HD HD NI NH NH NG NH NI NH HD NH NH NH NH HD and NI NH HD NG HD HD NH NG HD HD NG 
HD HD NH HD. Colony-PCR after the first TALEN reaction was conducted with the primers pCR8_F1 (5'-TTGATGCCTGGCAGTTCCCT-3') and pCR8_R1 (5'CGAACCGAACAGGCTTATGT-3'). After the second Golden Gate reaction the colony-PCR was performed with the primers TAL_F1 (5'TTGGCGTCGGCAAACAGTGG-3') and TAL_R2 (5'GGCGACGAGGTGGTCGTTGG-3').

iPSC Differentiation into mature, human, mid-brain specific dopaminergic neurons (hDANs)

hDANs were generated from iPSCs via neuropregenitor (NPC) intermediates using a protocol adapted from (Reinhardt, Glatza et al., 2013b). Briefly, IPSCs were maintained in E8 medium. For the generation of embryoid bodies (EBs), iPSCs were cultured in '50:50 base medium' (one to one mixture of DMEM Hams F12 (\#FG4815 Biochrome/Millipore): Neurobasal® medium (\#21103-049 Gibco/Thermo Scientific), 1X Penicillin/Streptomycin (Biochrome/Millipore), 1X GlutaMAX supplement Thermo Scientific), 1X B27 supplement (Gibco/Thermo Scientific) and 1X N2 supplement (Gibco/Thermo)) plus the addition of $10 \mu \mathrm{M}$ SB431542 (Sigma, SB), $1 \mu \mathrm{M}$

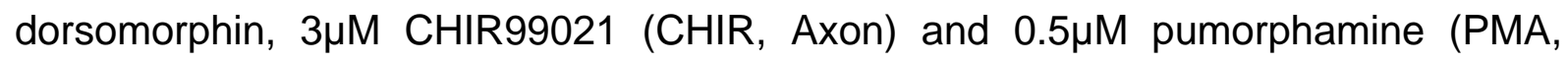
Alexis) on uncoated 6-well cell culture plates. EBs were then transferred to Matrigel (Corning)-coated 6-well plates in NPC maintenance media ('50:50 base medium' plus the addition of $150 \mu \mathrm{M}$ Ascorbic Acid (AA, Sigma), $3 \mu \mathrm{M}$ CHIR, 0.5 $\mu \mathrm{M}$ PMA). After several passages NPCs were cultivated in NPC priming medium (' $50: 50$ base medium' plus the addition of $150 \mu \mathrm{M}$ AA and $3 \mu \mathrm{M}$ CHIR99021). Differentiation of confluent NPCs was initiated by cultivation in a patterning medium for seven days ('50:50 base medium' plus the addition of $10 \mathrm{ng} / \mathrm{mL}$ FGF8 (Peprotech), $1 \mu \mathrm{M}$ PMA, $200 \mu \mathrm{M}$ AA, 20ng/mL BDNF (Peprotech). The differentiating neurons were matured in 
maturation media ('50:50 base medium' plus the addition of $10 \mathrm{ng} / \mathrm{mL}$ BDNF, 10ng/mL GDNF (Peprotech), 1ng/mL TGFß-III (Peprotech), 200 $\mu \mathrm{M}$ AA, $500 \mu \mathrm{M}$ dbcAMP (Applichem) and 10 MM DAPT (Sigma). Prior to all mitochondrial function experiments (ATP assay, measurement of reactive oxygen species, MitoTimer, respiratory measurements, complex I activity assay, glutathione assay, lipid peroxidation, apoptosis, LDH release and mitochondrial membrane potential), maturation medium was replaced $24 \mathrm{~h}$ before the experiment with 'N2 medium' (using 50:50 base media without B27 supplement or ascorbic acid) to reduce excessive amounts of antioxidants in the media that could quench oxidative stress or respiratory phenotypes.

\section{Animal work}

Pink1-knock-out mice (Glasl et al., 2012) with 4-16 months of age were used and bred in accordance with the regulations from the government of Upper Bavaria. The mice are on kept on a C57BL/6J background. All mice were kept on an inverse 12-h light/12-h dark cycle (lights off at 18:00). Mice were provided with ad libitum access to standard chow and water. Mice were killed by cervical dislocation and the brain immediately removed. Thereafter single brain regions were dissected and the tissue shock frozen in liquid nitrogen.

\section{Quantitative Reverse Transcription PCR (qRT-PCR)}

RNA was isolated from neurons using a RNeasy Mini Kit (QIAGEN), including the oncolumn DNA digestion step. A one-step qRT-PCR was performed on $0.1-1 \mu \mathrm{g}$ RNA (equalized to the same imput amount) with the QuantiTect SYBR Green RT-PCR Kit (QIAGEN) on a LightCycler $\AA_{480}$ (Roche). The relative expression levels were calculated with the $2^{-\Delta}$ method, based on a biological reference and housekeeping 
genes (GAPDH and HMBS) for normalization. The following primer sequences were used

\section{Immunofluorescence staining}

hDANs were cultivated on Matrigel-coated glass coverslips and fixed with 4\% (w/v) paraformaldehyde (PFA, Sigma) in phosphate buffered saline (PBS) for $15 \mathrm{~min}$ at room temperature $(\mathrm{RT})$. Permeabilisation with ice-cold, neat methanol for $5 \mathrm{~min}$ at $20^{\circ} \mathrm{C}$. After washing with $0.01 \%(\mathrm{v} / \mathrm{v})$ Tween in PBS (PBS-T), the fixed hDANs were blocked with $5 \%(\mathrm{v} / \mathrm{v})$ normal goat serum in PBS-T for $1 \mathrm{~h}$ at RT. Afterwards the cells were washed again and incubated overnight at $4^{\circ} \mathrm{C}$ with the primary antibody in $2.5 \%$ $(\mathrm{v} / \mathrm{v})$ serum in PBS-T. On the following day the cells were incubated with the secondary fluorescent antibody (\#A32721, \#A11070, \#A21449, \#A11010 all from Molecular probes/Thermo Scientific) for $2 \mathrm{~h}$ at RT in darkness and nuclei stained with DAPI (Sigma). Coverslips were mounted in mounting medium (Dako) on glass slides. Immunofluorescence was imaged using an AxioVert fluorescence microscope (Zeiss). MAP2 (AbCam ab5392) ( $n=$ diff5), TH (AbCam ab112) ( $n=$ diff5), FOXA2 (Millipore AB4125) (ndiff 3), DAT (Millipore MAB369) (nDiff3).

\section{LDH Assay}

LDH release was measured using the Pierce LDH Cytotoxicity Assay kit (Thermo Scientific). hDANs were seeded on a Matrigel coated 96 well plate. hDANs were treated in triplicates with and without $50 \mathrm{nM}$ and $100 \mathrm{nM}$ rotenone in $\mathrm{N} 2$ medium for $24 \mathrm{~h}$ at $37^{\circ} \mathrm{C}$ with $5 \% \mathrm{CO}_{2} .110 \mu \mathrm{L}$ of the celcu I Iture medium was transferred to an empty 96-well plate to obtain a spontaneous release fraction. Lysis buffer was added to the cell plate and then both 96 -well plates were incubated for 45 minutes at $37^{\circ} \mathrm{C}$ with $5 \% \mathrm{CO}_{2}$. Both plates were centrifuged for $5 \mathrm{~min}$ at $3220 \mathrm{rpm}$ at RT. $30 \mu \mathrm{L}$ 
sample from each cell line, each condition and each technical replicate was transferred to a 96-well ELISA reader plate (Cellstar) and the reaction started, measured and stopped according to the manufacturer's recommendations. LDH activity was calculated by subtraction of the $680 \mathrm{~nm}$ absorbance from the $490 \mathrm{~nm}$ absorbance values. Cytotoxicity was determined by dividing the LDH activity from the spontaneous release fractions by total LDH activity from the lysed samples. Increased LDH release caused by cell damage and apoptosis.

\section{SDS-PAGE and Western blotting}

All cell lysates were prepared in RIPA buffer (Sodium chloride 150mM, Tris-HCL $50 \mathrm{mM}$, Sodium dodecyl sulfate $0.10 \%(\mathrm{w} / \mathrm{v})$, Sodium deoxycholate $0.50 \%(\mathrm{w} / \mathrm{v})$, Triton-X-100 1\% (v/v)) containing $1 \mathrm{X}$ concentration of phosphatase inhibitor cocktail (Complete, Roche) and phosphatase inhibitor cocktail (PhosSTOP, Roche). Briefly, the lysis buffer was added directly to washed cells in dishes or washed cell pellets and kept at $4{ }^{\circ} \mathrm{C}$. Needles were used to further homogenize the lysates ( 9 passes 20G, 9 passes $27 G$ ) and incubated 30 minutes on ice. Insoluble nuclear material was removed after centrifugation at $14,000 \mathrm{rpm}$ for 10 minutes. Proteins were electrophoresed on self-made acrylamide gels or pre-cast Bis-Tris gels (Thermo Scientific) and transferred to nitrocellulose membranes using the iBlot device (Thermo Scientific), with the exception of very large target proteins or heavily lipidated proteins, in which case wet blotting with PVDF membranes were used. Total protein stain Ponceau (Applichem) was used to assess transfer and loading and the PageRuler plus pre-stained protein ladder (Thermo Scientific) for kDa range. Antibodies against mAconitase (BD Biosciences), $\beta$-Actin (Sigma Aldrich), GAPDH (Invitrogen, Thermo Scientific) Tom20 (Santa Cruz Biotechnology), LC3I/II (Novus), PGC1a (AbCam), Miro1 (Sigma Aldrich), OPA1 (Novus Biologicals), TH (Millipore), 
Flotilin (Santa Cruz), Vinculin (Sigma-Aldrich), Rab8A (Cell Signaling Technology),

HtrA2 (R\&D Systems), ATP5A (AbCam), DAT (Millipore), P-Thr53 DAT (Thermo Fisher), vGLUT1 (Stressmarq), MFRN2 (Thermo Fisher), GRP75 (Santa Cruz), GAP43 (Novus) and PGRMC1 (Proteintech), ERK1/2 and P-ERK1/2 (Cell Signaling Technolgy) and mitobiogenesis antibody (containing SDH, GAPDH and COX, AbCam) were used. Secondary antibodies were purchased from GE Healthcare (HRP-conjugated) and from LiCOR ( $\alpha$-rabbit and mouse Alexa Fluophor ${ }^{\mathrm{TM}} 680$, $\alpha-$ rabbit and mouse Alexa Fluophor ${ }^{\mathrm{TM}} 800$ ). Fluorescence detection and analysis were performed using a LI-CORE blot scanner and Image Studio ${ }^{\mathrm{TM}}$ Lite software. Densitometry from Western blot was performed using the Image J 1.410 software (Wayne Rasband; National Institutes of Health, USA).

\section{Autophagy and mitophagy induction}

Autophagic flux was induced in hDANs using $50 \mathrm{mM}$ ammonium chloride for $1 \mathrm{~h}, 1 \mu \mathrm{M}$ Valinomycin for 24h. Mitophagy was induced in hDANs using; $10 \mu \mathrm{M} \mathrm{CCCP} \mathrm{for} 2 \mathrm{~h}$, $4 \mathrm{~h}, 6 \mathrm{~h},(6 \mathrm{~h}+10 \mu \mathrm{M} M \mathrm{M} 132)$ and $24 \mathrm{~h}$ or $1 \mu \mathrm{M}$ Valinomycin for $24 \mathrm{~h}$.

\section{Mitochondrial turnover using MitoTimer ${ }^{\mathrm{TM}}$}

Mitotimer is a fluorescent reporter suitable for the investigation of mitochondrial turnover. Based on oxidation state, the fluorescent protein (dsred mutant) targeted to the mitochondria shifts its emission spectra from green to red as the protein matures. 98. Approximately 100,000 hDANs were seeded per 24-well well on coated coverslips in maturation medium.

For the analysis the IMARIS software was used. hDANs were transfected with pMitoTimer a gift from Zhen Yan (Addgene plasmid \# 52659 ; http://n2t.net/addgene:52659 ; RRID:Addgene_52659) using Fugene HD (Promega) 
according to manufacturer's instructions. The cells were fixed 8,24 and $48 \mathrm{~h}$ posttransfection with 4\% (v/v) PFA in PBS for 10 min RT. Transfected neurons were imaged for both green (Ex/Em, 488nm/518nm) and red channels (Ex/Em, 543nm/572nm) using a LSM 510 confocal (Zeiss). Z-Stack pictures were analyzed using Imaris 8.3.1 (Bitplane). The interpretation of the red:green MitoTimer ratio as an indication of mitochondrial turnover was according to PMID:4128932.

\section{Respiratory analyses}

For the basic mitochondrial stress test, OCR and ECAR were measured in NPCs and hDANs using a Seahorse ${ }^{\mathrm{TM}}$ XF96 Extracellular Flux Analyzer. 24 h. Cells were seeded in Matrigel coated Seahorse cell plates 24-48h prior to the experiment. During the experiemnet cells were treated sequentially with $1 \mu \mathrm{M}$ Oligomycin (Santa

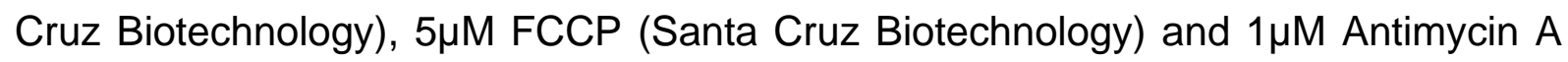
(Santa Cruz Biotechnology)/ $1 \mu \mathrm{M}$ Rotenone (Sigma) in order to perform a mitochondrial stress test where the base medium contained glucose, pyruvate and glutamine. Normalisation was performed by counting the number of cells per well of the plate using a high content imager from BD Biosciences. Following the respiratory analysis, the media is removed and the cells are fixed with $4 \%(w / v)$ PFA containing Hoescht stain $(1: 10,000)$ for 5 minutes. Then washed and stored in PBS for imaging.

For the Fatty Acid Oxidation (FAO) test, OCR and ECAR were measured in hDANs seeded on Matrigel coated seahorse cell plates 24-48h prior to the experiment with 30,000-40,000 cells per well. Briefly, hDAN media was exchanged for FAO media (Seahorse base medium containing 2.5mM glucose, 5mM HEPES, 0.5mM Carnitine, $\mathrm{pH7.4).} \mathrm{After} 20$ minutes incubated in a non-CO2 incubator, BSA, or BSA-Palmitate was added to half of the plate in the presence or absence of Etomoxir (Eto) before starting the assay. Measurements were made in the basal sate before injection of 
oligomycin, followed by FCCP and finally rotenone plus antimycin $A$ as in the mitochondrial stress test above.

\section{Flow cytometry experiments}

hDANs were carefully washed in PBS and then treated with Accumax to remove them from the monolayer, quenched in PBS and centrifuged at $300 \mathrm{~g}$ for 5 minutes and then incubated in dye, buffer only or dye plus a control.

For early apoptosis; Annexin V-Pacific blue in Annexin V binding buffer (both from BioLegend) or Annexin V-Pacific blue plus Staurosporine was used.

For mitochondrial membrane potential, $200 \mathrm{nM}$ Tetramethylrhodamine, Ethyl Ester, Perchlorate (TMRE, from Thermo Scientific) in PBS or TMRE plus Carbonyl cyanidep-trifluoromethoxyphenylhydrazone (CCCP) $10 \mu \mathrm{M}$ was used.

For mitochondrial reactive oxygen species, $2 \mu \mathrm{M}$ MitoSox ${ }^{\mathrm{TM}}$ (Thermo Scientific) in PBS or MitoSox ${ }^{\mathrm{TM}}$ plus $10 \mu \mathrm{M}$ Rotenone was used.

Cells were measured using a MACSQuant ${ }^{\circledR}$ automated flow cytometer (Mitenyi Biotechnology) according to their mean average fluorescence signal. All mean average fluorescence values were divided by the background fluorescence in the same channel in the same unstained cells to account for auto-fluorescence.

\section{Live Cell Kinetic Measurement of Mitochondrial Membrane Potential}

Cells were seeded in Ibidi® dishes and the media exchanged for HBSS containing $200 \mathrm{nM}$ TMRE stain (Thermo Scientific) for 15 minutes at $37^{\circ} \mathrm{C}$ with $\mathrm{CO}_{2}$. The TMRE was removed and replaced with $360 \mu$ l Hanks buffer. The cells were imaged using a Zeiss Inverted Confocal microscope at Excitation HeNe1, $543 \mathrm{~nm}$ and Emission LP $560 \mathrm{~nm}$ and brightfield for $20 \times 4$ s cycles. Followed by the addition of $360 \mu \mathrm{L}(0.25$ 
$\mathrm{mg} / \mathrm{ml}$ Oligomycin), measured for $20 \times 4$ s cycles, $180 \mu \mathrm{L}(10 \mu \mathrm{M}$ Rotenone $)$, measured for $20 \times 4$ s cycles and $100 \mu \mathrm{L}(10 \mu \mathrm{M}$ FCCP $)$ and measured for $20-40 \times 4 \mathrm{~s}$ cycles. Using Image J, each transfected cell (detected using ZsGreen-TRAP1) in each frame was analysed for TMRE fluorescence intensity, mean fluorescence and total area. The corrected total cell fluorescence (CTCF) over time was calculated using the formula: CTCF $=$ fluorescence intensity-(cell area $\times$ mean background fluorescence).

\section{RNA sequencing}

Approximately 5 million hDANs were lysed in 350ml RTL buffer and homogenized using QIAshredder® homogenizer (Qiagen). RNA isolation was performed using RNeasy Mini Kit (Qiagen). RNA was eluted in 30 $\mathrm{ll}$ RNase-free water. RNA quality was assessed with an Agilent 2100 Bioanalyzer and the Agilent RNA 6000 Nano kit (Agilent). Samples with very high RNA integrity number (RIN > 9) were selected for library construction. For polyA enrichment, a total of 200 ng of total RNA was subjected to polyA enrichment and cDNA libraries were constructed using the resulting mRNA and the Illumina TruSeq Stranded mRNA kit (Illumina). Libraries were sequenced as single reads (65 bp read length) on a HighSeq 2500 (Illumina) with a depth of $>22$ million reads each.

Library preparation and sequencing procedures were performed by the same individual and a design aimed to minimize technical batch effects was chosen.

Read quality of raw RNA-seq data in FASTQ files was assessed using QoRTs (v1.2.37) to identify sequencing cycles with low average quality, adaptor contamination, or repetitive sequences from PCR amplification. Reads were aligned using STAR (v2.5.3a) allowing gapped alignments to account for splicing to the 
Ensembl Homo sapiens GRCh37 reference genome. Alignment quality was analyzed using ngs-bits (v0.1) and visually inspected with the Integrative Genome Viewer (v2.4.19). Normalized read counts for all genes were obtained using subread (v1.5.1) and edgeR (v3.24.3). Transcripts covered with less than 1 count-per-million in at least 5 out of 6 sample were excluded from the analysis leaving $>13,000$ genes for determining differential expression in each of the pair-wise comparisons between experimental groups.

Pathway Analysis: CPM data were refined by log2 fold change values (logFC) of PINK1 KO hDANs compared to control hDANs. We used Ingenuity Pathways Analysis (IPA, QIAGEN). First, twenty genes with the highest and lowest log2 fold change were listed. Based on terms 'Parkinson's disease', 'dopamine metabolism' and known PINK1 interactions, several hit genes were validated in three independent differentiations by qRT-PCR. of sequencing data have been validated via quantitative reverse transcription-PCR (qRT-PCR). Interesting genes have been selected by the following criteria: a high logFC in sequencing sets, have a CMPvalues $>5$ and occur in both PINK1 KO hDAN clones.

\section{Phosphate-affinity polyacrylamide gel electrophoresis (PhosTag SDS-PAGE)}

For Parkin decorated Phos-Tag acrylamide gels, $100 \mu \mathrm{mol}$ Phos-Tag (WAKO Chemicals) and $200 \mu \mathrm{mol} \mathrm{MnCl} 2$ were added to the SDS-PAGE and EDTA-free Laemmli buffer was used for sample loading. For all other experiemnts gels were prepared using $7.5 \%(\mathrm{v} / \mathrm{v})$ polyacrylamide gel with $25 \mu \mathrm{M}$ Phos-tag and $50 \mu \mathrm{M} \mathrm{MnCl} 2$ or Zinc-pre-cast PhosTag gels. Note that the prestained molecular weight markers contain EDTA and therefore do not resolve at the exact kDa on PhosTag gels. All PhoTag gels were run at $\sim 30 \mathrm{~V}$ or slower. Phosphorylation-dependent mobility shifts of some proteins are dramatically improved by lowering the current. All gels were 
gently agitated in transfer buffer supplemented with $1 \mathrm{mM}$ EDTA for $10 \mathrm{~min}$ then washed for 10 minutes in the transfer buffer alone before transfer. We used iBlot semi-dry blotting with nitrocellulose. Blocking, incubation with antibodies, and detection are according to standard Western blotting procedures.

\section{Phosphoproteomics}

Samples preparation for mass spectrometry: Proteins from crude mitochondrial enrichment lysates were precipitated using acetone precipitation as described in ("Maximizing recovery of water-soluble proteins through acetone precipitation"; Crowell, Wall and Doucette; Analytic Chimica Acta 2013). Acetone precipitated proteins were denatured in urea buffer $(6 \mathrm{M}$ urea, $2 \mathrm{M}$ thiourea in $10 \mathrm{mM}$ Tris buffer, $\mathrm{pH}$ 8.0) and protein concentration was determined by applying Bradford assay (BioRad). Disulfide bonds were reduced by $10 \mathrm{mM}$ of dithiothreitol (DTT) for $1 \mathrm{~h}$ at RT under agitation at $800 \mathrm{rpm}$. In a next step alkylation with $50 \mathrm{mM}$ iodoacetamide (IAA) was performed for $1 \mathrm{~h}$ at RT under agitation in the dark. Subsequent protein digestion in a ratio of $1 \mu \mathrm{g}$ enzyme per $100 \mu \mathrm{g}$ protein for $3 \mathrm{~h}$ with the endoproteinase Lys-C, and overnight with trypsin was performed. To stop the enzymatic reaction and to load peptides onto C18-material for purification, samples were acidified to $\mathrm{pH} 2.5$ with trifluoroacetic acid (TFA).

For quantitative analysis samples were on-column (SepPak C18, Waters) labeled with dimethyl as described previously (Boersema, Nature Protocols volume 4, pages 484-494 (2009)). In more detail, two triple dimethyl sets were prepared, combining either the untreated or the simvastatin treated samples. Primary amines of peptides derived from WT were labeled with the light, $\mathrm{KO} 1$ with the medium and $\mathrm{KO} 2$ with the heavy labeled version of formaldehyde. Label efficiency of each dimethyl channel and the correct 1:1:1 mixing of each triple dimethyl set was validated by a pilot LC- 
MS/MS measurement. All label efficiencies were above $99 \%$ and the mixing correction factors for the two dimethyl sets were applied based on the label mixing ratios. Labeled peptides were eluted from the column by $80 \%$ acetonitrile (ACN). This left around $1.5 \mathrm{mg}$ of labeled peptide mixture for the enrichment of phosphorylated peptides and $20 \mu \mathrm{g}$ for the analysis of the proteome. Proteomic samples were subjected to purification by C18 StageTips as described previously (Ishihama, J. Proteome Res.200654988-994). Three consecutive rounds of phosphopeptide enrichment were performed by using MagReSyn®Ti-IMAC beads (ReSynBiosiences) according to the manufactures instruction. For all enrichment rounds $1 \mathrm{mg}$ magnetic Ti-IMAC beads per $500 \mu \mathrm{g}$ protein digest were used. Eluted peptides were purified by C18 StageTips prior to LC-MS/MS based analysis.

Mass spectrometry: Proteome and Phosphoproteome samples were measured on a Q Exactive ${ }^{\mathrm{TM}}$ HF Hybrid Quadrupol-Orbitrap instrument, online connected to an Easy1200 nanoflow HPLC system (Thermo Fischer Scientific).

The liquid chromatography-Mass spectrometry (LC-MS) analysis was performed as described in Schmitt et al https://doi.org/10.1074/mcp.RA119.001302. Briefly, the proteome samples were separated in a 230 min gradient of the binary solvent system compromising of solvent $A$ and $B$ on an in-house packed $20 \mathrm{~cm}$ reverse-phase $\mathrm{C} 18$ (1.9 $\mu \mathrm{m}$; Dr. Maisch) nano-HPLC column. The phopsproteome samples were run in a 60 min gradient. The following instrument settings were used for the quantitative phosphoproteome analysis: full MS scans were recorded with a resolution of 60,000. The 7 most intense ions were selected for higher-energy collisional dissociation (HCD) fragmentation and ions were recorded with a resolution of 60,000 . For the proteome analysis the 12 most intense peptides were selected and analyzed with a resolution of 30,000 . 
MS raw data were, processed by using Andromeda integrated in MaxQuant 1.5.5.1 (Cox J. Proteome Res.20111041794-1805; Cox Nat Protoc. 2009; 4(5):698-705) with default settings. Peak lists were searched against the target decoy UniProt human database (release 2019_02_13; 74,468 proteins. For quantification, the dimethylation on lysine residues and $\mathrm{N}$ termini was defined as "light labels" (+ $28 \mathrm{Da})$, "medium labels" (+ $32 \mathrm{Da})$, and "heavy labels" (+ $36 \mathrm{Da})$. The match-between-runs option between consecutive phosophoproteomic data was enabled. Oxidation of methionine and phosphorylation of serine $(\mathrm{S})$, threonine $(\mathrm{T})$ and tyrosine $(\mathrm{Y})$ were set as variable modifications.

For a more accurate quantification the dimethyl ratios of identified phosphorylation events were normalized to the ratios of the corresponding protein groups by using Perseus software (version 1.5.3.2). Significantly regulated phosphorylation events log2-transformed dimethyl ratios were computed by the Significance B test and a pvalue of 0.05 .

\section{Crude mitochondrial enrichment}

Crude mitochondrial enrichment: Fresh cell pellets on ice were resuspended in mitochondrial isolation buffer (10 mM HEPES pH 7.4, $50 \mathrm{mM}$ sucrose, $0.4 \mathrm{M}$ mannitol, $10 \mathrm{mM} \mathrm{KCl}, 1 \mathrm{mM}$ EGTA, phosphatase and protease inhibitors (Roche)) and passed through 20G, 27G and 30G needles 8 times to disrupt cells. Fractionation was then achieved by several centrifugation steps. Fist, samples were centrifuged for $5 \mathrm{~min}$ at $1000 \mathrm{xg}$ at $4^{\circ} \mathrm{C}$ and the supernatant was saved. The pellet was resuspended in $500 \mu \mathrm{L}$ mitochondrial isolation buffer and passed through a 30G needle 8 times before centrifuging $5 \mathrm{~min}$ at $1000 \mathrm{xg}$ at $4^{\circ} \mathrm{C}$. The second supernatant was pooled with the first and centrifuged for $15 \mathrm{~min}$ at $15,000 \mathrm{xg}$ at $4^{\circ} \mathrm{C}$. The obtained pellet comprises the mitochondrial fraction and was resuspended in $300 \mu \mathrm{L}$ 
PBS containing 1\% TritonX-100, phosphatase and protease inhibitors. Mitochondria were lysed for 15 min on ice with repetitive vortexing.

\section{Cholesterol Assay}

The Total Cholesterol Assay Kit (Fluorometric) (STA-390, Cell Biolabs Inc) was performed according to manufacturer's instructions. Absolute cholesterol levels were measured using cholesterol standards and normalized to protein input in the lipid extraction.

\section{Lipidomics}

Lipidomics of whole hDANs from independent differentiations $(n=3)$ were performed according to the exact method described in ${ }^{99}$.

Lipidomics of crude mitochondrial preparations from hDANs from independent differentiations $(n=3)$ were performed according to the methods described in ${ }^{100,101}$.

\section{Sucrose fractionation of hDANs and depletion of cholesterol}

Detergent solubilisation of hDANs and subsequent sucrose gradient fractionation was performed as described in ${ }^{92}$. The 14 fractions were stored at $-80{ }^{\circ} \mathrm{C}$ and then proteins were precipitated using the methanol-chloroform method for all fractions to concentrate the sample sufficiently to detect proteins in the higher fractions.

\section{BRET Assay Mito-ER Tethering}

All constructs for expression using Sindbis virus, were synthetized in pSinRep5 (Thermo Fisher Scientific). Restriction enzymes Mlu1 and Stul were used for the insertion of the constructs into the SR5 vector. Sindbis virus containing BRET biosensor (Donor: mitochondria targeted Renilla luciferase, Rluc and Acceptor: ER- 
targeted mVenus) were produced following protocol described in ${ }^{102}$, with minor modifications. Briefly, BHK-21 cells were co-transfected with pSinRep5 RNA of interest and helper pDHtRNA. After 48 hours, viruses were purified by sucrose gradient centrifugation. Samples were centrifuged 90 minutes at $35.000 \mathrm{rpm}\left(4^{\circ} \mathrm{C}\right)$ in a SW 60 Ti swinging-bucket rotor (Beckman Coulter) in a Beckman Optima L-100K. Viral particles were collected from the $20 \% / 55 \%$ sucrose interface. NPCs and hDANS were infected with sindbis virus containing BRET biosensor for $48 \mathrm{~h}$. Cells were washed with PBS, incubated with $5 \mu \mathrm{M}$ Coelentrazine h (Promega) in PBS for 10min in the dark. BRET measurements were carried out in a Tecan Infinite M200 plate reader at RT. BRET signal was calculated as acceptor emission relative to donor emission and corrected by subtracting the background ratio value detected when Rluc is expressed alone.

\section{Dynamic light scattering (DLS)}

Dynamic light scattering is a technique used in physics for size characterization of proteins, nanoparticles, polymers and colloidal dispersions. hDANs were washed in PBS and suspended in $350 \mu$ l of a standard HPLC elution buffer that does not contain detergents (see HPLC analysis). The hDAN suspension were homgenised using $5 \mathrm{~mm}$ stainless steel beads and the tissue lyser LT (Qiagen) for 4 minutes with $50 \mathrm{~Hz}$.To remove large impurities the homogenized cell suspension was filtered through a $0.2 \mu \mathrm{m}$ nylon membrane. The filtered suspension was then diluted in water and run through a Malvern DLS instrument at $21^{\circ} \mathrm{C}$. Particle size $(\mathrm{nm})$ and sample polydispersity index (Pdi) are calculated automatically and analyses were made according to the manufacturer's instructions. Samples with Pdi values close to 1 or 1 are generally considered too heterogeneous for the calculation of particle size and 
therefore these measuremenet were removed from the mean size data but not the mean Pdi data. $n=$ diff 3 .

\section{Neurotransmitter Uptake Assay}

Neurotransmitter transporter activity in hDANs was measured using the Neurotransmitter Transporter Uptake Assay Kit (Molecular Devices) according to the manufacturer's instructions. Mature hDANs were seeded in Matrigel coated black, clear bottom 96 -well plates prior to the assay at a density of 60,000 /well in triplicates. Wells containing no cells were used as an internal control. hDANs were treated with a specific VMAT2 inhibitor (Tetrabenazine, 10 $\mathrm{MM}$ ), a TH inhibitor (3-londol-LTyrosine, $300 \mu \mathrm{M}$ ), or a combination of MAO and COMT inhibitors (Tranylcypromine $10 \mu \mathrm{M}$ and Tolcapone 100nM respectively). These compounds were added in media containing L-DOPA $(50 \mu \mathrm{M})$ or without L-DOPA. A subset of hDANs triplicates were treated with media alone or media containing L-DOPA $(50 \mu \mathrm{M})$. All treatments were incubated on the cells at $37^{\circ} \mathrm{C}$ for 20 minutes prior to the addition of the substrates. Uptake fluorescence was measured using the SpectraMax $\mathrm{M}_{2}{ }^{\mathrm{e}}$ microplate reader in kinetic mode (Molecular Devices) measuring every 30s and using SoftMaxPro 6.4 Software (Molecular Devices) for detection. After the assay, the hDANs were washed and fixed in $4 \%(\mathrm{v} / \mathrm{v})$ PFA containing Hoescht to account for cell number in each well and then counterstained for $\mathrm{TH}$ to account for any large differences in $\mathrm{TH}$ positive hDANs in the culture wells. Statistical analyses were accomplished by the unpaired two-sided Student's t-test $\left({ }^{*} \mathrm{p}<0.05,{ }^{* *} \mathrm{p}<0.01\right)$.

\section{Dopamine (Catecholamine) oxidation assay}


The catecholamine oxidation assay was performed according to ${ }^{103}$ using Biodyne® B $0.45 \mu \mathrm{m}$ membranes (Pall corporation) and detection using a LiCOR fluorescent scanning device.

\section{Monoamine oxidase (MAO) Activity Assay}

MAO activity was monitored using a radiometric assay with 14C-tyramine hydrochloride as substrate as previously described ${ }^{104}$. Data were normalized for protein content and rates expressed as disintegrations of $14 \mathrm{C} / \mathrm{min} / \mu \mathrm{g}$ protein.

\section{Electrophysiology}

Whole-cell patch clamp experiments were performed at room temperature using an Axopatch 200B amplifier (Molecular devices). Data were low-pass filtered at $10 \mathrm{kHz}$, digitized at $10 \mathrm{kHz}$ via a Digidata $1440 \mathrm{~A}$ acquisition system and analyzed offline using Clampfit 10.7 (Molecular Devices, Sunnyvale, CA, USA). During electrophysiological recordings, cells were kept in an extracellular solution (5mM HEPES (pH 7.4), $140 \mathrm{mM} \mathrm{NaCl}, 4.2 \mathrm{mM} \mathrm{KCl,} 1 \mathrm{mM} \mathrm{MgSO} 4.7 \mathrm{H} 2 \mathrm{O}, 1.1 \mathrm{mM}$ $\mathrm{CaCl} 2.2 \mathrm{H} 2 \mathrm{O}, 0.5 \mathrm{mM}$ Na2HPO4, $0.45 \mathrm{mM}$ NaH2PO4, $10 \mathrm{mM}$ glucose). Patch pipettes were pulled from borosilicate glass (Science products) using a Sutter P97 Puller (Sutter Instruments Company). Their resistance ranged from 3 to $5 \mathrm{M} \Omega$. Patch pipettes were filled with intracellular solution (10 mM HEPES (pH 7.2), 5 mM EGTA, 135 mM K-gluconate, 4 mM NaCl, 0.5 mM CaCl2, 2 mM ATP-Mg, 0.4 mM GTP-Na) and an osmolarity of 290 mOsm. Series resistance (<20 M $\Omega$ ) was monitored during the experiment. Cells showing unstable series resistance or resting membrane potential were discarded. Liquid junction potential was not subtracted from the data and it was calculated as $-15 \mathrm{mV}$. The data are shown with leak subtraction. Voltageclamp experiments were performed to record inward and outward currents generated 
via depolarization pulses from a holding potential of $-70 \mathrm{mV}$ to different potential levels, from $-60 \mathrm{mV}$ to $+30 \mathrm{mV}$ with $10 \mathrm{mV}$ increments, for 24 milliseconds. To assess intrinsic firing properties, current-clamp experiments were performed holding the neurons at $55 \mathrm{mV}$ level via continuous current injection and responses to the stepwise current stimulations from $-0.001 \mathrm{nA}$ to $0.069 \mathrm{nA}$ in 0.005 increments with a duration of 800 ms were recorded.

\section{Calcium imaging}

Mature neurons were seeded on Matrigel-coated glass coverslips. Fluo-4 Direct Calcium Reagent (Invitrogen) was added to the cells and incubated for one hour at $37^{\circ} \mathrm{C}$ and $5 \% \mathrm{CO}_{2}$. After washing the cells with growth medium, the coverslips were transferred into the imaging chambers and Fluo-4 reagent diluted in growth medium was added. 3 mM EGTA was added per well 10 minutes prior to imaging. Neurons were imaged on a LSM 510 Confocal microscope (Zeiss) taking a picture every 0.5 sec for 25 minutes. A baseline was recorded for two minutes and then $2 \mu \mathrm{M}$ Thapsigargin was added. The data analysis was performed using ImageJ. The corrected total cell fluorescence per cell was determined and plotted over time to evaluate the calcium content.

\section{Protein estimation}

The protein content of cell supernatants was determined with a Pierce ${ }^{\mathrm{TM}}$ BCA Protein Assay Kit (ThermoFisher) and normalized to $20-80 \mu \mathrm{g}$ protein per lane depending on abundance.

\section{Mitochondrial Morphology (BacMam Mitogreen)}

Neurons were plated on Matrigel covered glass coverslips, treated with CellLight ${ }^{\mathrm{TM}}$ Mitochondria-GFP, BacMam 2.0 (Invitrogen) according to manufacturer's instructions 
for 24 hours in N2 medium. Mitochondria of transfected neurons were imaged using an LSM-510 confocal microscope (Zeiss). Z-stack pictures were analysed with ImageJ, to determine the aspect ratio, form factor, area and circularity of mitochondria according to ${ }^{105}$.

\section{Kinetic analysis of mitochondrial membrane potential}

Neurons on Matrigel covered glass coverslips were treated with N2 medium $24 \mathrm{~h}$ prior to the experiment. Kinetic TMRE measurements were conducted as described $^{106}$ and imaged with a LSM-510 confocal microscope (Zeiss). The corrected total cell fluorescence was determined using ImageJ.

\section{Analysis of cellular ROS}

Medium was changed to N2 medium $24 \mathrm{~h}$ prior to the experiment. On the following day neurons were incubated with $\mathrm{N} 2$ medium or $\mathrm{N} 2$ medium containing $10 \mu \mathrm{M}$ Rotenone for $4 \mathrm{~h}$ before measurement. $100 \mu \mathrm{M}$ dihydroethidium (DiHET) (Santa Cruz Biotechnology) was added to all wells and fluorescence emission at $420 \mathrm{~nm}$ (cytosolic DiHET, non-oxidized) and 610nm (chromatin-bound, oxidized DiHET) was measured every 30 secs for $30 \mathrm{~min}$ on a SpectraMax® M microplate reader (Molecular Devices).

\section{GSH assessment}

Neurons on a 96-well plate were treated with different treatment conditions and N2 medium $24 \mathrm{~h}$ prior to the experiment. Cells were briefly washed with HBSS (Gibco)

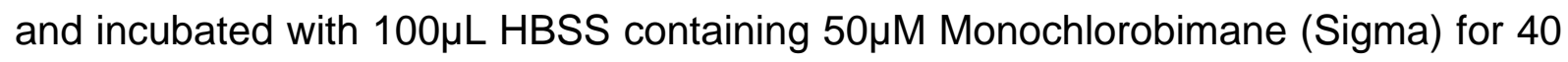
minutes @37 ${ }^{\circ} \mathrm{C}, 5 \% \mathrm{CO}$. After washing with HBSS, cells were imaged with a SpectraMax® M microplate reader (Molecular Devices) (Ex 390nm/Em 478nm). 


\section{Aconitase activity}

Aconitase activity was measured in whole cell lysates from neurons using an adapted protocol $^{107}$. Absorbance at $340 \mathrm{~nm}$ was measured using a SpectraMax® M microplate reader (Molecular Devices).

\section{NAD ${ }^{+}$and NADH levels}

Neurons were cultivated in N2 medium $24 \mathrm{~h}$ before the experiment. Whole cell NAD+and NADH-levels were determined using the NAD/NADH Assay Kit (Fluorometric) from Abcam according to manufacturer's instructions. NADH reaction mixture was incubated for 1 hour and fluorescence $(E x / E m=540 / 590 \mathrm{~nm})$ was measured using a SpectraMax® M microplate reader (Molecular Devices).

\section{Complex I and citrate synthase activity}

Complex I activity was measured in crude mitochondrial enrichments from hDANs previously described in ${ }^{105}$. Following isolation of crude mitochondria, a total protein content $>0.7 \mathrm{mg} / \mathrm{mL}$ is required to have enough active mitochondria for each sample in triplicate in the assay and again duplicated for the rotenone negative control. Complex I activity was measured according to ${ }^{108}$. The activity of complex I was normalized to citrate synthase activity, also according to ${ }^{108}$ and data expressed as a ratio of complex $\mathrm{l} / \mathrm{citrate}$ synthase.

\section{Transmission Electron microscopy}

Neurons were seeded on Matrigel (Corning) coated glass coverslips and cultivated for three days prior to treatment with $\mathrm{N} 2$ medium with and without $1 \mu \mathrm{M}$ Valinomycin for 24 hours. After washing and fixation with $2.5 \%$ glutaraldehyde (Science Services, Munich, Germany) in cacodylate buffer (pH7.4; Merck-Millipore, Darmstadt, 
Germany) overnight at $4^{\circ} \mathrm{C}$, cells were washed with cacodylate buffer, post fixed in $1 \%$ osmiumtetroxide, dehydrated and embedded in epoxide resin (Araldite, Serva, Heidelberg, Germany) as described previously ${ }^{109}$. Ultrathin sections were performed using a Reichert Ultracut ultramicrotome (Leica, Bensheim, Germany) and were analyzed in an EM 10 electron microscope (Zeiss, Oberkochen, Germany). Images were taken by a digital camera (Tröndle, Germany).

\section{Measurement of biogenic amines by HPLC-ED}

Mature hDANs were expanded during differentiation into $175 \mathrm{~cm}^{2}$ Matrigel coated flasks. For each experiment, approximately $2-3175 \mathrm{~cm}^{2}$ flasks were required. Although endogenous dopamine levels could be detected in hDANs derived from much larger cell volumes, we employed 50 $\mathrm{MM}$ L-DOPA treatment overnight (16h) to enhance dopamine metabolism without risk of dopamine toxicity (see ${ }^{103}$ and ${ }^{110}$ ). Following detachment of hDANs with Accumax, the cell suspensions were washed in PBS and cells counted. PBS was used to normalise the number of cells in the suspension. An aliquot of the normalized suspension was taken for preparation of protein lysate and RNA to determine the relative amount of $\mathrm{TH}$ positive cells in each experiment and differentiation for each cell line. Fresh (unfrozen) cell pellets kept on ice were suspended in $350 \mu$ l of a standard HPLC elution buffer that does not contain detergents (Thermo Scientific). The suspensions were homogenized using $5 \mathrm{~mm}$ stainless steel beads and the tissue lyser LT (Qiagen) for 4 minutes with $50 \mathrm{~Hz}$.To remove large impurities the homogenized cell suspension was filtered through a $0.2 \mu \mathrm{m}$ nylon membrane. Samples were analysed for catecholamine and indolamine content by ion-pair reverse phase HPLC with coulometric detection (Ultimate 3000 LC with electrochemical detection ECD3000RS, Thermo Fischer Scientific, California, USA). A hypersil C18 colum was used $(150 \times 3 \mathrm{~mm}, 3 \mu \mathrm{m})$ and the system was run 
with a Test mobild phase containing $10 \%$ actonitril and $1 \%$ phosphate buffer (Thermo Fischer Scientific, California, USA) at a flow rate of $0.4 \mathrm{ml} / \mathrm{min}$ at $30{ }^{\circ} \mathrm{C}$. The potential of the first channel was set to $+350 \mathrm{mV}$, the second channel to $-250 \mathrm{mV}$.

Epinephrine, Norepinephrine, Dopamine, 3,4-dihydroxyphenylacetic acid (DOPAC), homovanillic acid (HVA), 5-hydroxyindol-acetic acid (HIAA), 3-Methoxythyramine (3MT) and serotonin (5-HT), concentration was determined by comparing peak areas of the samples with those of standards using Chromeleon 7 chromatography data system software. The neurochemicals in standards were determined with a high correlation linearity $(r 2=0.98)$ and good reproducibility in retention time $(0.03 \%)$. The limit of detection was $<1 \mathrm{pg}$ on column for all the metabolites analyzed.

\section{Complex I Dipstick Assay}

Active Complex I was pulled down from hDAN homogenates using the Complex I

Dipstick Assay from AbCam (ab109720) according to the manufacturer's instructions.

\section{Mitochondrial Import Assay}

Radiolabeled proteins were synthesized in rabbit reticulocyte lysate in the presence of 35S-methionine after in vitro transcription by SP6 polymerase from pGEM4 vectors (Promega). Radiolabeled precursor proteins were incubated at either $30^{\circ} \mathrm{C}$ (pSu9DHFR) or $4^{\circ} \mathrm{C}$ (Fis 1 ) in import buffer (250 mM sucrose, $0.25 \mathrm{mg} / \mathrm{ml} \mathrm{BSA}, 80 \mathrm{mM}$ $\mathrm{KC1}, 5 \mathrm{mM} \mathrm{MgCl}, 10 \mathrm{mM}$ MOPS-KOH, 2 mM NADH, $4 \mathrm{mM}$ ATP, $\mathrm{pH}$ 7.2) with mitochondria isolated from the indicated cells. Non-imported pSu9-DHFR was removed by treatment with proteinase $\mathrm{K}(\mathrm{PK}, 50 \mu \mathrm{g} / \mathrm{ml})$ for $30 \mathrm{~min}$ on ice and then PK was inhibited with $5 \mathrm{mM}$ PMSF. Membrane integration of Fis1 molecules was confirmed by resistance to alkaline extraction (incubation on ice for 30 min with $0.1 \mathrm{M}$ 
Na2CO3). Finally, all the samples were boiled at $95^{\circ} \mathrm{C}$ for few min before their analysis by SDS-PAGE.

\section{Acknowledgements}

The German Research Foundation and the research training group MOMbrane and The German Federal Ministry of Education (BMBF) 'Mito PD' were the primary source of funding and allowed collaborations that made this work possible. Funded by the German Research Foundation (DFG), GRK2364.

Maria Calleja-Felipe of the Molecular Cognition Laboratory, Biophysics Institute, Consejo Superior de Investigaciones Cientificas (CSIC)-University of the Basque Country (UPV)/Euskal Herriko University (EHU), Campus Universidad del País Vasco, 48940 Leioa, Spain.and, Shira Knafo of the Molecular Cognition Laboratory, Biophysics Institute, Consejo Superior de Investigaciones Cientificas (CSIC)University of the Basque Country (UPV)/Euskal Herriko University (EHU), Campus Universidad del País Vasco, 48940 Leioa, Spain. Ikerbasque, Basque Foundation for Science, 48013 Bilbao, Spain. Department of Physiology and Cell Biology and National Institute of Biotechnology in the Negev, Faculty of Health Sciences, BenGurion University of the Negev, Beer-Sheva, 8410501 Israel who developed the constructs for performing the BRET experiments for measurement of mitochondrialER contacts and kindly shared them with HFR and AG. Gerrit Machetanz of the Hertie Institute for Clinical Brain Research, The University of Tübingen, University Clinic Tübingen for clinical assistance and discussions concerning PINK1 Parkinson's disease. 


\section{Author contributions}

1) Research project: A. Conception (JCF, TG, PK), B. Organization (JCF, SG, RK, TG, PK, AG, BM, HK, DVW, WW), C. Execution (JCF, CB, BB, NC, PFB, MF, HFR, SG, AS, BS, LS, AUK, BU, MZ, KZ, DVW).

2) Statistical Analysis: A. Design (JCF, CB, AS, MF, NC, PFB, SG, KZ, BM, BB, HFR, HK, DR) B. Execution (JCF, CB, AS, MF, NC, PFB, SG, KZ, BM, HFR, HK) C. Review and Critique; (JCF, SG, PK, TG, MF, AS, NC, AG, HFR, HK, AUK, KZ, BM, DVW, WW, DR)

3) Manuscript: A. Writing of the first draft (JCF), B. Review and Critique (DVW, JCF, SG, RK, TG, AG, MF, HFR, PK, AUK, KZ, BM, WW).

\section{Conflict of Interest}

The authors declare no conflict of interest.

\section{The paper explained}

Problem. Parkinson's disease is a common neurodegenerative disease that is currently incurable. There is little known about why dopamine-containing neurons are so vulnerable. It is not clear how mitochondrial defects (that occur in all body cell types) cause neuronal dysfunction and neuronal death in Parkinson's disease.

Results. We studied dopaminergic neurons grown from healthy and Parkinson's disease stem cells. Mitochondrial vulnerability induced by defective PINK1 (a Parkinson's disease gene) is compensated for in maturing human neurons and mitochondrial networks are normal. A compensatory metabolic shift provides lipids for piecemeal renewal of mitochondrial membranes in the absence of PINK1-dependent mitophagy. However, these biochemical events have a negative impact at mitochondrial-ER contact sites (affecting central metabolism and dopamine metabolism) and at the plasma membranes making them more ridged and therefore more susceptible to synaptic defects (including dopamine uptake).

Impact. These data highlight the complexity and difficulties in treating Parkinson's disease. Here the biological mechanism uncovered is both necessary for the survival of neurons during development but leads to their vulnerability in aging. We propose 
that low doses of compounds such as $\beta$-cyclodextrin which make cell membranes more fluid could benefit patients by treating the secondary (but more specific) cause of disease rather than the initial defect which has so far proven unsuccessful.

\section{Data availability}

- RNA Seq data: All raw data and log2 values for the genome.

- Phospho-proteome data: All raw data and phosphorylation sites of all unfiltered and filtered data.

- Lipidomics: All lipidomics data.

Links to Excel and Text files will be made via https://datadryad.org/stash and a link via https://www.hih-tuebingen.de/en/forschung/neurodegenerativediseases/research-groups/mitochondrial-biology-of-parkinsons-disease/

The data will be uploaded on datadryad if this manuscript is accepted for peer review so it is accessible for reviewers.

\section{Figure Legends}

Figure. 1 A) Scheme showing removal of PINK1 exon 1 (homozygous) using TALEN gene editing in healthy iPSCs. Sequence confirmation of two clonal lines. B) Percent of hDANs in a field of view positive for MAP2, FOXA2, TH and DAT using immunocytochemistry. Each point on the graph is a technical replicate from four independent differentiations (ndiff=4). C) Relative gene expression of neuronal markers in PINK1 KO hDANs compared to the healthy control. Each point on the graph is the mean average gene expression from RNA isolated from an independent hDAN differentiation. D) Western blots showing autophagic flux (LC3-II accumulation c.a LC3-I, Aconitase and $\beta$-actin) in untreated hDANs and those stimulated by valinomycin (Val, $1 \mu \mathrm{M}, 24 \mathrm{~h})$ or ammonium chloride $(\mathrm{NH} 4 \mathrm{Cl}, 50 \mathrm{mM}, 1 \mathrm{~h})$ representative blots (ndiff=3). E) Western blots of PINK1-Parkin pathway proteins in 
hDANs untreated or following $10 \mu \mathrm{M}$ CCCP treatment for $0,2,6$ and $24 \mathrm{~h}-/+$

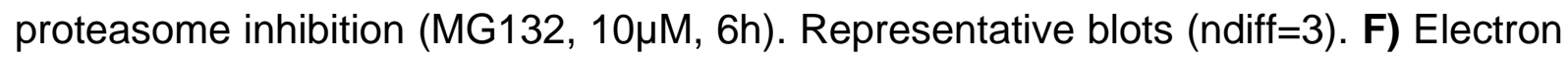
microscope images of hDANs untreated or treated with valinomycin (Val, $1 \mu \mathrm{M}, 24 \mathrm{~h})$. Representative images (ndiff=3). G) Quantification of Western blots for PGC1a (as a ratio to cytosolic marker GAPDH, left panel) and nuclear encoded SDH (as a ratio to mitochondrially expressed MTCO1, middle panel) (ndiff=3). Quantification of mitochondrial renewal measured by the amount of expressed Fis MitoTimer construct (red) as a ratio to oxidised Fis (green) where oxidation represents time present (right panel). Quantification of live cell microscopy images was performed blinded using Imaris software (ndiff=4).

Figure 2 A) Respiratory analyses of oxygen consumption (left panel) and extracellular acidification rate (middle panel) during mitochondrial stress for hDANs where glucose and pyruvate is given and oxygen consumption (right panel) during mitochondrial stress in hDANs where carnitine and glucose is given with or without the exogenous fatty acid palmitate (Palm). B) NADH dehydrogenase (complex I) enzyme activity in enriched mitochondrial fractions from neurons where NADH and ubiquinone is given, $\mathrm{NADH}$ depletion rate in the presence of rotenone is accounted for and then normalised to citrate synthase activity as a marker of mitochondrial mass ( $n=$ diff5). C) Citrate synthase enzyme activity in enriched mitochondrial fractions from hDANs and normalised to total protein concentration of the mitochondrial preparation ( $n=$ diff3). D) Mitochondrial membrane potential measured by TMRM fluorescence quantified from live cell images per cell over a time course of where oligomycin, Rotenone and then FCCP are sequentially added ( $n=$ diff3). E) Mitochondrial reactive oxygen species (Mito ROS) measured using MitoSox fluorescence by flow cytometry 
with or without acute rotenone treatment to generate ROS. Mean fluorescence values were normalised to the background fluorescence of the same hDANs without the dye and normalised to the untreated control ( $n=$ diff3). F) Aconitase enzyme activity in hDANs normalised to cell number and healthy control ( $n=$ diff3). G) NAD concentration of hDANs normalised to total protein and to the healthy control ( $n=$ diff5). H) Pathway analysis of RNAseq data from hDANs where each pathway shows log2 gene expression level comparing genotype in untreated hDANs (CTRL $v$ $\mathrm{KO}$ ), effect of $24 \mathrm{~h}$ ionophore treatment on control hDANs (CTRL v Val), effect of $24 \mathrm{~h}$ ionophore treatment on PINK1 KO hDANs (KO v Val) and genotype plus treatment change (Gen v Val). I) Free cholesterol, total cholesterol and cholesterol ester levels in hDANs normalised to total protein levels ( $n=$ diff3). J) Lipidomics performed on enriched mitochondrial fractions from hDANs where the percent lipid content is normalised to the healthy control for cholesterols (left panel), cholesteryl ester (middle panel) and cardiolipin (right panel) ( $\mathrm{n}=$ diff 3 ). K) Lipidomics performed on whole hDANs where the percent lipid content is shown for those lipid species downregulated in both PINK1 KO clones (left panel) and those lipid species upregulated in both PINK1 KO clones (right panel) compared to control. Two way ANNOVA comparing genotype and lipid.

Figure 3 A) Mitochondrial-ER tethering (Mito-ER tethering) in NPC neuroprogenitors and hDANs measured by BRET signal which is increased as mitochondria and endoplasmic reticulum (ER) are closer in proximity $(n=3$ NPC independent experiments and hDAN differentiations). B) Cytosolic calcium levels in response to the addition of thapsigargin during live cell imaging measured by quantifying Fluo4 dye fluorescence in hDANs. The total corrected cell fluorescence (accounting for cell area) was normalised in each image to the baseline measurement before addition of 
thapsigargin after incubation with EGTA ( $n=$ diff4). C) Increased mitoferrin 2 gene expression (left panel) and protein abundance (right panel) normalised to GAPDH ( $n=$ diff3). D) Time course of Miro1 phosphorylation status following 10 $\mu \mathrm{M}$ CCCP to stabilise PINK1 and MG132 to block Miro1 degradation using PhosTag SDS-PAGE (representative blots, $n=$ diff3). E) Phosphorylation status of Miro1 in NPC neuroprogenitors and hDANs where CCCP is used to depolarise the MOM and stabilise PINK1 (representative blots, $n=$ diff3). F) Plot of the most differentially phosphorylated proteins in enriched mitochondrial fractions from hDANs untreated or treated with Simvastatin. Log2 ratio of phosphor-proteins is normalised to the total proteome in each case ( $\mathrm{n}=\operatorname{diff} 1)$. G) Mitoferrin 2 gene expression and protein levels relative to GAPDH ( $n=$ diff3) (upper panel). Phosphorylation status of tyrosine hydroxylase $(\mathrm{TH})$ in NPC neuroprogenitors and hDANs where CCCP is used to depolarise the MOM and stabilise PINK1 (representative blots, n=diff3).

Figure 4 A) Properties of particles in hDAN homogenates; dispersity (heterogeneity of shape and size) index measured by dynamic light scattering (upper panel) where a poly dispersity index (Pdi) of 1.0 reflects a homogenate that contains extremely heterogeneously sized particles not considered suitable for particle size measurement. Particle size was calculated for those homogenates with a Pdi of 0.75 or under using dynamic light scattering ( $n=$ diff3). B) Sucrose gradient fractionation of total hDAN lysates followed by protein precipitation and SDS PAGE to detect markers of the soluble and lipid raft floating fractions (representative blots, $n=d i f f 3$ ). C) Graph showing the amount of quantified flotillin (lipid raft marker) and ERK1/2 (cytosolic marker) distribution across the 14 fractions. D) Distribution of dopamine transporter (DAT) and Phospho-DAT (P-DAT Thr53) throughout the hDAN fractions ( $\mathrm{n}=$ diff3). E) Acute $1 \mathrm{mM}$ B-cyclodextrin treatment depletes cholesterol and solubilises 
marker proteins in hDANs ( $\mathrm{n}=$ diff3). F) Chronic 500nM B-cyclodextrin treatment of hDANs during neuronal maturation restores mitochondrial membrane potential measured by TMRE fluorescence using flow cytometry ( $n=$ diff6). G) Chronic 500nM B-cyclodextrin treatment of hDANs during neuronal maturation restores oxygen consumption (respiratory) response to utilise exogenous fatty acids as an energy source.

Figure 5 A) Concentration of neurotransmitters and metabolites epinephrine (E), 3,4Dihydroxyphenylacetic acid (DOPAC), Dopamine (DA), 5-Hydroxyindoleacetic acid (HIAA), Homovanillic acid (HVA), 3-Methoxytyramine (3-MT) and 3-(2-aminoethyl)-1 $\mathrm{H}$-indol-5-ol /Serotonin (5-HT) in hDANs treated with L-DOPA for $24 \mathrm{~h}(\mathrm{n}=\mathrm{diff5})$. B) Ratios of DOPAC/DA, HVA/DA and DOPAC plus HVA/DA. C) Tyrosine hydroxylase (TH) protein levels (left panel) and gene expression (right panel) in hDAN aliquots from each experiment/hDAN differentiation shown in A) and B). D) Dopamine concentration in hDANs and in cell culture supernatants treated with L-DOPA for $24 \mathrm{~h}$ with or without inhibition of dopamine degradation via COMT and MAOA/B (MOA/COMT inhib') n=diff3). E) MAO A/B activity in hDANs measured by the deamination of radiolabelled tyramine (left panel) and MAO-A protein levels normalised to GAPDH marker and the healthy control (right panel) ( $\mathrm{n}=\mathrm{diff} 3)$. F) Catecholamine oxidation in hDANs with or without L-DOPA treatment for $24 \mathrm{~h}$ (representative blots, $\mathrm{n}=$ diff3). G) Neurotransmitter uptake in hDANs measured by the fluorescence of the labelled amine converted inside hDANs only ( $n=$ diff3). G) Quantification of neurotransmitter uptake in hDANs with or without acute inhibition of TH activity (THi), inhibition of VMAT2 activity (VMAT2i), inhibition of COMT and MAOA/B activity (COMTi/MAOi) with or without $24 \mathrm{~h}$ L-DOPA treatment. The rate of uptake is normalised to the amount of $\mathrm{TH}$ staining in the well to account for hDAN 
number ( $\mathrm{n}=$ diff3). I) Trace activity of (TH positive) hDANs patched and unstimulated (two upper panels) and PINK1 KO hDANs following hyperpolarisation ( $\mathrm{n}=$ diff3). J) Resting membrane potential (upper panel) and amplitude (lower panel) of hDANs $(n=\operatorname{diff3})$.

Supplementary Figure S1A) Relative PINK1 gene expression in control and PINK1 KO hDAN clones using primers directed to exon1-4 and exon 2-4 ( $n=$ diff3). S1B) Immunocytochemistry staining of MAP2, TH and FOXA2 in fixed hDANs. Representative images are shown ( $\mathrm{n}=$ diff4). S1C) Representative images of $\mathrm{Cl}$ dipstick assay for active $\mathrm{Cl}$ in hDANs treated with or without $1 \mu \mathrm{M}$ valinomycin for $24 \mathrm{~h}$ (left panel). Quantification of dipstick band from three independent experiments ( $n=$ diff3). S1D) Mean average TMRE fluorescence signal during flow cytometry for untreated hDANs and those treated with $10 \mu \mathrm{M}$ CCCP in vitro. The signal is normalised to the unstained signal from the same cells. $n=$ diff 3 . S1E) Kinetic measurement of dihydroethidium-cytosolic ROS over time in the presence or absence of $1 \mathrm{mM}$ rotenone. S1F) Lipid peroxidation levels measured using BODIPY in hDANs treated with rotenone, L-DOPA, DA and 6-hydroxydopamine (6-ODHA) for 24h. S1G) Glutathione assay in hDANs in the presence or absence of rotenone, glutathione depleting BSO or both together. $\mathrm{n}=$ diff3. $\mathbf{S 1 H}$ ) alpha-ketoglutarate dehydrogenase enzyme activity in hDANs. $n=$ diff3. S1I) Total NADH levels in hDANs. $\mathrm{n}=$ diff3.

Supplementary Figure 2A) Heatmap of strongest log2 fold changes in gene expression between control and PINK1 KO genotypes in hDANS ( $n=3$, diff=1). S2B) $\%$ mol lipid of all measured lipid species from lipidomics of mitochondrial fractions of hDANs ( $\mathrm{n}=$ diff3). S2C) \% mol lipid of all measured lipid species from lipidomics of whole hDAN preparations ( $\mathrm{n}=$ diff3). S2D) Co-localisation (Pearson coefficient) of 
SREB to nuclei in fixed hDANs $(n=3)$. S2E) Readouts of mitochondrial morphology in hDANs from live cell imaging ( $\mathrm{n}=$ diff3). S2F) Phosphorylation status of TOM70, Mitoferrin2 (MFRN2) and GRP75 in NPC neuroprogenitors and hDANs where CCCP is used to depolarise the MOM and stabilise PINK1 (representative blots, $n=$ diff3). S2G) List of most regulated (log2 fold change) phospho-peptide abundance in hDANs untreated or treated with $5 \mu \mathrm{M}$ Simvastatin for $24 \mathrm{~h}$. The list was filtered and weighted for the lowest deviation between samples and *represents the fold change is in the same direction and ** reversed by Simvastatin. S2H) Phosphorylation status of GAP43 and PDRMC1 in hDANs where CCCP is used to depolarise the MOM and stabilise PINK1 (representative blots, $\mathrm{n}=$ diff3).

Supplementary Figure 3A) Relative Rab3B gene expression and 3B) Relative PNPO gene expression in NPC neuroprogenitors and hDANs ( $n=$ diff 3 ). 3C) Cholesterol levels measured in the ventral midbrain (VM, left) and striatum (STR, right) of WT (CTRL) and PINK1 KO (KO) mice at 4 months (young) or 16 months (old) of age. $\mathrm{n}=3$ animals per group. Mean average cholesterol $+/$ - standard deviation. ${ }^{*}=<0.05$, Student's T-test. 3D) Representative SDS PAGE Western blot images of mitochondrial protein import for pSU9-DHFR and Fis-1 (left) and quantification of SDS-PAGE Western blot images from four independent differentiations and experiments on fresh mitochondria (right). 
1. Gandhi S, Plun-Favreau H. Mutations and mechanism: how PINK1 may contribute to risk of sporadic Parkinson's disease. Brain. 2017 Jan;140(1):2-5.

2. Klein C, Grunewald A, Hedrich K. Early-onset parkinsonism associated with PINK1 mutations: frequency, genotypes, and phenotypes. Neurology. 2006 Apr 11;66(7):1129-30; author reply -30.

3. Schiesling C, Kieper N, Seidel K, Kruger R. Review: Familial Parkinson's disease--genetics, clinical phenotype and neuropathology in relation to the common sporadic form of the disease. Neuropathol Appl Neurobiol. 2008 Jun;34(3):255-71.

4. Valente EM, Abou-Sleiman PM, Caputo V, et al. Hereditary early-onset Parkinson's disease caused by mutations in PINK1. Science. 2004 May 21;304(5674):1158-60.

5. Steinlechner S, Stahlberg J, Volkel B, et al. Co-occurrence of affective and schizophrenia spectrum disorders with PINK1 mutations. J Neurol Neurosurg Psychiatry. 2007 May;78(5):532-5.

6. Esposito G, Vos M, Vilain S, et al. Aconitase causes iron toxicity in Drosophila pink1 mutants. PLoS Genet. 2013 Apr;9(4):e1003478.

7. Gautier CA, Kitada T, Shen J. Loss of PINK1 causes mitochondrial functional defects and increased sensitivity to oxidative stress. Proc Natl Acad Sci U S A. 2008 Aug 12;105(32):11364-9. 8. Gispert S, Ricciardi F, Kurz A, et al. Parkinson phenotype in aged PINK1-deficient mice is accompanied by progressive mitochondrial dysfunction in absence of neurodegeneration. PLoS One. 2009;4(6):e5777.

9. Heeman B, Van den Haute C, Aelvoet SA, et al. Depletion of PINK1 affects mitochondrial metabolism, calcium homeostasis and energy maintenance. J Cell Sci. 2011 Apr 1;124(Pt 7):1115-25. 10. Julienne H, Buhl E, Leslie DS, Hodge JJL. Drosophila PINK1 and parkin loss-of-function mutants display a range of non-motor Parkinson's disease phenotypes. Neurobiol Dis. 2017 Aug;104:15-23.

11. Moisoi N, Fedele V, Edwards J, Martins LM. Loss of PINK1 enhances neurodegeneration in a mouse model of Parkinson's disease triggered by mitochondrial stress. Neuropharmacology. 2014 Feb;77:350-7.

12. Parganlija D, Klinkenberg M, Dominguez-Bautista J, et al. Loss of PINK1 impairs stress-induced autophagy and cell survival. PLoS One. 2014;9(4):e95288.

13. Requejo-Aguilar R, Lopez-Fabuel I, Fernandez E, Martins LM, Almeida A, Bolanos JP. PINK1 deficiency sustains cell proliferation by reprogramming glucose metabolism through HIF1. Nat Commun. 2014;5:4514.

14. Villa E, Proics E, Rubio-Patino C, et al. Parkin-Independent Mitophagy Controls Chemotherapeutic Response in Cancer Cells. Cell Rep. 2017 Sep 19;20(12):2846-59.

15. Vives-Bauza C, Zhou C, Huang Y, et al. PINK1-dependent recruitment of Parkin to mitochondria in mitophagy. Proc Natl Acad Sci U S A. 2010 Jan 5;107(1):378-83.

16. Anichtchik O, Diekmann H, Fleming A, Roach A, Goldsmith P, Rubinsztein DC. Loss of PINK1 function affects development and results in neurodegeneration in zebrafish. J Neurosci. 2008 Aug 13;28(33):8199-207.

17. Soman $\mathrm{S}$, Keatinge M, Moein M, et al. Inhibition of the mitochondrial calcium uniporter rescues dopaminergic neurons in pink1(-/-) zebrafish. Eur J Neurosci. 2017 Feb;45(4):528-35.

18. Kitada T, Pisani A, Porter DR, et al. Impaired dopamine release and synaptic plasticity in the striatum of PINK1-deficient mice. Proc Natl Acad Sci U S A. 2007 Jul 3;104(27):11441-6.

19. Clark IE, Dodson MW, Jiang C, et al. Drosophila pink1 is required for mitochondrial function and interacts genetically with parkin. Nature. 2006 Jun 29;441(7097):1162-6.

20. Park J, Lee SB, Lee S, et al. Mitochondrial dysfunction in Drosophila PINK1 mutants is complemented by parkin. Nature. 2006 Jun 29;441(7097):1157-61.

21. Geisler S, Holmstrom KM, Skujat D, et al. PINK1/Parkin-mediated mitophagy is dependent on VDAC1 and p62/SQSTM1. Nat Cell Biol. 2010 Feb;12(2):119-31.

22. Narendra D, Tanaka A, Suen DF, Youle RJ. Parkin is recruited selectively to impaired mitochondria and promotes their autophagy. Journal of Cell Biology. 2008 Dec 1;183(5):795-803.

23. Narendra DP, Jin SM, Tanaka A, et al. PINK1 is selectively stabilized on impaired mitochondria to activate Parkin. PLoS Biol. 2010 Jan;8(1):e1000298. 
24. Jin SM, Lazarou M, Wang C, Kane LA, Narendra DP, Youle RJ. Mitochondrial membrane potential regulates PINK1 import and proteolytic destabilization by PARL. J Cell Biol. 2010 Nov 29;191(5):933-42.

25. Jin SM, Youle RJ. The accumulation of misfolded proteins in the mitochondrial matrix is sensed by PINK1 to induce PARK2/Parkin-mediated mitophagy of polarized mitochondria. Autophagy. 2013 Nov 1;9(11):1750-7.

26. Kane LA, Lazarou M, Fogel Al, et al. PINK1 phosphorylates ubiquitin to activate Parkin E3 ubiquitin ligase activity. Journal of Cell Biology. 2014 Apr 28;205(2):143-53.

27. Kazlauskaite A, Kondapalli C, Gourlay R, et al. Parkin is activated by PINK1-dependent phosphorylation of ubiquitin at Ser(65). Biochemical Journal. 2014 May 15;460:127-39.

28. Koyano F, Okatsu K, Kosako $\mathrm{H}$, et al. Ubiquitin is phosphorylated by PINK1 to activate parkin. Nature. 2014 Jun 5;510(7503):162-+.

29. Gegg ME, Cooper JM, Chau KY, Rojo M, Schapira AHV, Taanman JW. Mitofusin 1 and mitofusin 2 are ubiquitinated in a PINK1/parkin-dependent manner upon induction of mitophagy. Human Molecular Genetics. 2010 Dec 15;19(24):4861-70.

30. Birsa N, Norkett R, Wauer T, et al. Lysine 27 Ubiquitination of the Mitochondrial Transport Protein Miro Is Dependent on Serine 65 of the Parkin Ubiquitin Ligase. Journal of Biological Chemistry. 2014 May 23;289(21):14569-82.

31. Wang XN, Winter D, Ashrafi G, et al. PINK1 and Parkin Target Miro for Phosphorylation and Degradation to Arrest Mitochondrial Motility. Cell. 2011 Nov 11;147(4):893-906.

32. Lazarou M, Sliter DA, Kane LA, et al. The ubiquitin kinase PINK1 recruits autophagy receptors to induce mitophagy. Nature. 2015 Aug 20;524(7565):309-+.

33. Wong YC, Holzbaur EL. Optineurin is an autophagy receptor for damaged mitochondria in parkin-mediated mitophagy that is disrupted by an ALS-linked mutation. Molecular Biology of the Cell. 2014 Dec;25.

34. Narendra DP, Kane LA, Hauser DN, Fearnley IM, Youle RJ. p62/SQSTM1 is required for Parkininduced mitochondrial clustering but not mitophagy; VDAC1 is dispensable for both. Autophagy. 2010 Nov 16;6(8):1090-106.

35. Okatsu K, Saisho K, Shimanuki M, et al. p62/SQSTM1 cooperates with Parkin for perinuclear clustering of depolarized mitochondria. Genes to Cells. 2010 Aug;15(8):887-900.

36. McWilliams TG, Prescott AR, Montava-Garriga L, et al. Basal Mitophagy Occurs Independently of PINK1 in Mouse Tissues of High Metabolic Demand. Cell Metab. 2018 Feb 6;27(2):439-49 e5.

37. Lee JJ, Sanchez-Martinez A, Zarate AM, et al. Basal mitophagy is widespread in Drosophila but minimally affected by loss of Pink1 or parkin. J Cell Biol. 2018 Mar 2.

38. Walsh TG, van den Bosch MTJ, Lewis KE, Williams CM, Poole AW. Loss of the mitochondrial kinase PINK1 does not alter platelet function. Sci Rep. 2018 Sep 26;8(1):14377.

39. Chu CT. Multiple pathways for mitophagy: A neurodegenerative conundrum for Parkinson's disease. Neurosci Lett. 2019 Apr 1;697:66-71.

40. Cummins N, Gotz J. Shedding light on mitophagy in neurons: what is the evidence for PINK1/Parkin mitophagy in vivo? Cell Mol Life Sci. 2018 Apr;75(7):1151-62.

41. Wang Y, Tang CY, Cai J, et al. PINK1/Parkin-mediated mitophagy is activated in cisplatin nephrotoxicity to protect against kidney injury. Cell Death \& Disease. 2018 Nov 1;9.

42. Guo T, Liu T, Sun Y, et al. Sonodynamic therapy inhibits palmitate-induced beta cell dysfunction via PINK1/Parkin-dependent mitophagy. Cell Death Dis. 2019 Jun 11;10(6):457.

43. Berthier A, Navarro S, Jimenez-Sainz J, et al. PINK1 displays tissue-specific subcellular location and regulates apoptosis and cell growth in breast cancer cells. Hum Pathol. 2011 Jan;42(1):75-87.

44. Blackinton JG, Anvret A, Beilina A, Olson L, Cookson MR, Galter D. Expression of PINK1 mRNA in human and rodent brain and in Parkinson's disease. Brain Res. 2007 Dec 12;1184:10-6.

45. Zhang Y, Chen K, Sloan SA, et al. An RNA-sequencing transcriptome and splicing database of glia, neurons, and vascular cells of the cerebral cortex. J Neurosci. 2014 Sep 3;34(36):11929-47. 
46. Seibler P, Graziotto J, Jeong H, Simunovic F, Klein C, Krainc D. Mitochondrial Parkin recruitment is impaired in neurons derived from mutant PINK1 induced pluripotent stem cells. J Neurosci. 2011 Apr 20;31(16):5970-6.

47. Hamasaki M, Furuta N, Matsuda A, et al. Autophagosomes form at ER-mitochondria contact sites. Nature. 2013 Mar 21;495(7441):389-93.

48. Gelmetti V, De Rosa P, Torosantucci L, et al. PINK1 and BECN1 relocalize at mitochondriaassociated membranes during mitophagy and promote ER-mitochondria tethering and autophagosome formation. Autophagy. 2017;13(4):654-69.

49. Torres-Odio S, Key J, Hoepken HH, et al. Progression of pathology in PINK1-deficient mouse brain from splicing via ubiquitination, ER stress, and mitophagy changes to neuroinflammation. J Neuroinflammation. 2017 Aug 2;14(1):154.

50. Celardo I, Costa AC, Lehmann S, et al. Mitofusin-mediated ER stress triggers neurodegeneration in pink1/parkin models of Parkinson's disease. Cell Death Dis. 2016 Jun 23;7(6):e2271.

51. McLelland GL, Goiran T, Yi W, et al. Mfn2 ubiquitination by PINK1/parkin gates the p97dependent release of ER from mitochondria to drive mitophagy. Elife. 2018 Apr 20;7.

52. Kane LA, Youle RJ. PINK1 and Parkin flag Miro to direct mitochondrial traffic. Cell. 2011 Nov $11 ; 147(4): 721-3$.

53. Grossmann D, Berenguer-Escuder C, Bellet ME, et al. Mutations in RHOT1 Disrupt Endoplasmic Reticulum-Mitochondria Contact Sites Interfering with Calcium Homeostasis and Mitochondrial Dynamics in Parkinson's Disease. Antioxid Redox Signal. 2019 Aug 21.

54. Shoshan-Barmatz V, Zalk R, Gincel D, Vardi N. Subcellular localization of VDAC in mitochondria and ER in the cerebellum. Biochim Biophys Acta. 2004 Jul 9;1657(2-3):105-14.

55. Gandhi S, Wood-Kaczmar A, Yao Z, et al. PINK1-associated Parkinson's disease is caused by neuronal vulnerability to calcium-induced cell death. Mol Cell. 2009 Mar 13;33(5):627-38.

56. Allen GF, Toth R, James J, Ganley IG. Loss of iron triggers PINK1/Parkin-independent mitophagy. EMBO Rep. 2013 Dec;14(12):1127-35.

57. Horowitz MP, Greenamyre JT. Mitochondrial iron metabolism and its role in neurodegeneration. J Alzheimers Dis. 2010;20 Suppl 2:S551-68.

58. Li C, Zhang Y, Cheng $X$, et al. PINK1 and PARK2 Suppress Pancreatic Tumorigenesis through Control of Mitochondrial Iron-Mediated Immunometabolism. Dev Cell. 2018 Aug 20;46(4):441-55 e8. 59. Ivatt RM, Sanchez-Martinez A, Godena VK, Brown S, Ziviani E, Whitworth AJ. Genome-wide RNAi screen identifies the Parkinson disease GWAS risk locus SREBF1 as a regulator of mitophagy. Proc Natl Acad Sci U S A. 2014 Jun 10;111(23):8494-9.

60. Chu CT, Ji J, Dagda RK, et al. Cardiolipin externalization to the outer mitochondrial membrane acts as an elimination signal for mitophagy in neuronal cells. Nat Cell Biol. 2013 Oct;15(10):1197-205. 61. Vos M, Geens A, Bohm C, et al. Cardiolipin promotes electron transport between ubiquinone and complex I to rescue PINK1 deficiency. J Cell Biol. 2017 Mar 6;216(3):695-708.

62. Chen BB, Coon TA, Glasser JR, et al. E3 ligase subunit Fbxo15 and PINK1 kinase regulate cardiolipin synthase 1 stability and mitochondrial function in pneumonia. Cell Rep. $2014 \mathrm{Apr}$ 24;7(2):476-87.

63. Zhuang Z, Hogan M, McCauley R. The in vitro insertion of monoamine oxidase B into mitochondrial outer membranes. FEBS Lett. 1988 Sep 26;238(1):185-90.

64. Zhuang ZP, Marks B, McCauley RB. The insertion of monoamine oxidase A into the outer membrane of rat liver mitochondria. J Biol Chem. 1992 Jan 5;267(1):591-6.

65. Dunkley PR, Bobrovskaya L, Graham ME, von Nagy-Felsobuki El, Dickson PW. Tyrosine hydroxylase phosphorylation: regulation and consequences. J Neurochem. 2004 Dec;91(5):1025-43. 66. Sliter DA, Martinez J, Hao L, et al. Parkin and PINK1 mitigate STING-induced inflammation. Nature. 2018 Sep;561(7722):258-62.

67. West AP, Khoury-Hanold W, Staron M, et al. Mitochondrial DNA stress primes the antiviral innate immune response. Nature. 2015 Apr 23;520(7548):553-7. 
68. Matheoud D, Cannon T, Voisin A, et al. Intestinal infection triggers Parkinson's disease-like symptoms in Pink1(-/-) mice. Nature. 2019 Jul;571(7766):565-9.

69. Reinhardt $\mathrm{P}$, Glatza $\mathrm{M}$, Hemmer K, et al. Derivation and expansion using only small molecules of human neural progenitors for neurodegenerative disease modeling. PLoS One. 2013;8(3):e59252.

70. Morais VA, Haddad D, Craessaerts K, et al. PINK1 loss-of-function mutations affect mitochondrial complex I activity via NdufA10 ubiquinone uncoupling. Science. $2014 \mathrm{Apr}$ 11;344(6180):203-7.

71. Pogson JH, Ivatt RM, Sanchez-Martinez A, et al. The complex I subunit NDUFA10 selectively rescues Drosophila pink1 mutants through a mechanism independent of mitophagy. PLoS Genet. 2014 Nov;10(11):e1004815.

72. Lai YC, Kondapalli C, Lehneck R, et al. Phosphoproteomic screening identifies Rab GTPases as novel downstream targets of PINK1. EMBO J. 2015 Nov 12;34(22):2840-61.

73. Kanerva K, Uronen RL, Blom T, et al. LDL Cholesterol Recycles to the Plasma Membrane via a Rab8a-Myosin5b-Actin-Dependent Membrane Transport Route. Dev Cell. 2013 Nov 11;27(3):249-62. 74. Karam CS, Javitch JA. Phosphorylation of the Amino Terminus of the Dopamine Transporter: Regulatory Mechanisms and Implications for Amphetamine Action. Adv Pharmacol. 2018;82:205-34. 75. Vaughan RA, Foster JD. Mechanisms of dopamine transporter regulation in normal and disease states. Trends Pharmacol Sci. 2013 Sep;34(9):489-96.

76. McWilliams TG, Prescott AR, Montava-Garriga L, et al. Basal Mitophagy Occurs Independently of PINK1 in Mouse Tissues of High Metabolic Demand. Cell Metab. 2018 Jan 10.

77. Wang X, Winter D, Ashrafi G, et al. PINK1 and Parkin Target Miro for Phosphorylation and Degradation to Arrest Mitochondrial Motility. Molecular Biology of the Cell. 2011;22.

78. Pridgeon JW, Olzmann JA, Chin LS, Li L. PINK1 protects against oxidative stress by phosphorylating mitochondrial chaperone TRAP1. PLoS Biol. 2007 Jul;5(7):e172.

79. Burman JL, Pickles S, Wang C, et al. Mitochondrial fission facilitates the selective mitophagy of protein aggregates. J Cell Biol. 2017 Oct 2;216(10):3231-47.

80. Pickles S, Vigie P, Youle RJ. Mitophagy and Quality Control Mechanisms in Mitochondrial Maintenance. Curr Biol. 2018 Feb 19;28(4):R170-R85.

81. Soubannier V, McLelland GL, Zunino R, et al. A vesicular transport pathway shuttles cargo from mitochondria to lysosomes. Curr Biol. 2012 Jan 24;22(2):135-41.

82. Rieusset J. The role of endoplasmic reticulum-mitochondria contact sites in the control of glucose homeostasis: an update. Cell Death Dis. 2018 Mar 9;9(3):388.

83. Saiki S, Hatano T, Fujimaki M, et al. Decreased long-chain acylcarnitines from insufficient beta-oxidation as potential early diagnostic markers for Parkinson's disease. Sci Rep. 2017 Aug 4;7(1):7328.

84. Lloyd T, Weisz J. Direct Inhibition of Tyrosine-Hydroxylase Activity by Catechol Estrogens. Journal of Biological Chemistry. 1978;253(14):4841-3.

85. Lloyd T, Weisz J, Breakefield XO. Catechol Estrogen, 2-Hydroxyestradiol, Inhibits Catechol-OMethyltransferase Activity in Neuroblastoma-Cells. Journal of Neurochemistry. 1978;31(1):245-50.

86. Allen GF, Neergheen V, Oppenheim M, et al. Pyridoxal 5'-phosphate deficiency causes a loss of aromatic L-amino acid decarboxylase in patients and human neuroblastoma cells, implications for aromatic L-amino acid decarboxylase and vitamin B(6) deficiency states. J Neurochem. 2010 Jul;114(1):87-96.

87. Tufi R, Gandhi S, de Castro IP, et al. Enhancing nucleotide metabolism protects against mitochondrial dysfunction and neurodegeneration in a PINK1 model of Parkinson's disease. Nat Cell Biol. 2014 Feb;16(2):157-66.

88. Guardia-Laguarta C, Area-Gomez E, Schon EA, Przedborski S. A new role for alpha-synuclein in Parkinson's disease: Alteration of ER-mitochondrial communication. Mov Disord. 2015 Jul;30(8):1026-33.

89. Jiang $\mathrm{H}$, Jiang $\mathrm{Q}$, Liu $\mathrm{W}$, Feng J. Parkin suppresses the expression of monoamine oxidases. J Biol Chem. 2006 Mar 31;281(13):8591-9. 
90. Hauptmann N, Grimsby J, Shih JC, Cadenas E. The metabolism of tyramine by monoamine oxidase A/B causes oxidative damage to mitochondrial DNA. Arch Biochem Biophys. 1996 Nov 15;335(2):295-304.

91. Kang SS, Ahn EH, Zhang Z, et al. alpha-Synuclein stimulation of monoamine oxidase-B and legumain protease mediates the pathology of Parkinson's disease. EMBO J. 2018 Jun 15;37(12).

92. Chamberlain LH, Burgoyne RD, Gould GW. SNARE proteins are highly enriched in lipid rafts in PC12 cells: implications for the spatial control of exocytosis. Proc Natl Acad Sci U S A. 2001 May 8;98(10):5619-24.

93. Guardia-Laguarta C, Coma M, Pera M, et al. Mild cholesterol depletion reduces amyloid-beta production by impairing APP trafficking to the cell surface. J Neurochem. 2009 Jul;110(1):220-30.

94. Zhang J, Liu Q. Cholesterol metabolism and homeostasis in the brain. Protein Cell. 2015 Apr;6(4):254-64.

95. Brakedal B, Haugarvoll K, Tzoulis C. Simvastatin is associated with decreased risk of Parkinson disease. Ann Neurol. 2017 Feb;81(2):329-30.

96. Reinhardt P, Schmid B, Burbulla LF, et al. Genetic correction of a LRRK2 mutation in human iPSCs links parkinsonian neurodegeneration to ERK-dependent changes in gene expression. Cell Stem Cell. 2013 Mar 7;12(3):354-67.

97. Cermak T, Doyle EL, Christian M, et al. Efficient design and assembly of custom TALEN and other TAL effector-based constructs for DNA targeting. Nucleic acids research. 2011 Jul;39(12):e82. 98. Hernandez G, Thornton C, Stotland A, et al. MitoTimer: a novel tool for monitoring mitochondrial turnover. Autophagy. 2013 Nov 1;9(11):1852-61.

99. Xicoy H, Brouwers JF, Kalnytska O, Wieringa B, Martens GJM. Lipid Analysis of the 6Hydroxydopamine-Treated SH-SY5Y Cell Model for Parkinson's Disease. Mol Neurobiol. 2019 Sep 6. 100. Chowdhury A, Aich A, Jain G, et al. Defective Mitochondrial Cardiolipin Remodeling Dampens HIF-1alpha Expression in Hypoxia. Cell Rep. 2018 Oct 16;25(3):561-70 e6.

101. Ozbalci C, Sachsenheimer T, Brugger B. Quantitative analysis of cellular lipids by nanoelectrospray ionization mass spectrometry. Methods Mol Biol. 2013;1033:3-20.

102. Malinow R, Hayashi $Y$, Maletic-Savatic $M$, et al. Introduction of green fluorescent protein (GFP) into hippocampal neurons through viral infection. Cold Spring Harb Protoc. 2010 Apr;2010(4):pdb prot5406.

103. Burbulla LF, Song P, Mazzulli JR, et al. Dopamine oxidation mediates mitochondrial and lysosomal dysfunction in Parkinson's disease. Science. 2017 Sep 22;357(6357):1255-61.

104. Ugun-Klusek A, Theodosi TS, Fitzgerald JC, et al. Monoamine oxidase-A promotes protective autophagy in human SH-SY5Y neuroblastoma cells through Bcl-2 phosphorylation. Redox Biol. 2019 Jan;20:167-81.

105. Fitzgerald JC, Zimprich A, Berrio DAC, et al. Metformin reverses TRAP1 mutation-associated alterations in mitochondrial function in Parkinson's disease. Brain. 2017 Sep;140:2444-59.

106. Plun-Favreau H, Burchell VS, Holmstrom KM, et al. HtrA2 deficiency causes mitochondrial uncoupling through the $F(1) F(0)$-ATP synthase and consequent ATP depletion. Cell Death Dis. 2012;3:e335.

107. Pierik AJ, Netz DJ, Lill R. Analysis of iron-sulfur protein maturation in eukaryotes. Nat Protoc. 2009;4(5):753-66.

108. Hargreaves IP, Duncan AJ, Wu L, Agrawal A, Land JM, Heales SJ. Inhibition of mitochondrial complex IV leads to secondary loss complex II-III activity: implications for the pathogenesis and treatment of mitochondrial encephalomyopathies. Mitochondrion. $2007 \mathrm{Jul} ; 7(4): 284-7$.

109. Wolburg-Buchholz K, Mack AF, Steiner E, Pfeiffer F, Engelhardt B, Wolburg H. Loss of astrocyte polarity marks blood-brain barrier impairment during experimental autoimmune encephalomyelitis (vol 118, pg 219, 2009). Acta Neuropathol. 2009 Oct;118(4):583-.

110. Allen GF, Ullah Y, Hargreaves IP, Land JM, Heales SJ. Dopamine but not I-dopa stimulates neural glutathione metabolism. Potential implications for Parkinson's and other dopamine deficiency states. Neurochem Int. 2013 Apr;62(5):684-94. 
bioRxiv preprint doi: https://doi.org/10.1101/814343; this version posted October 22, 2019. The copyright holder for this preprint (which was not certified by peer review) is the author/funder, who has granted bioRxiv a license to display the preprint in perpetuity. It is made

Bus et al. Figure 2

A
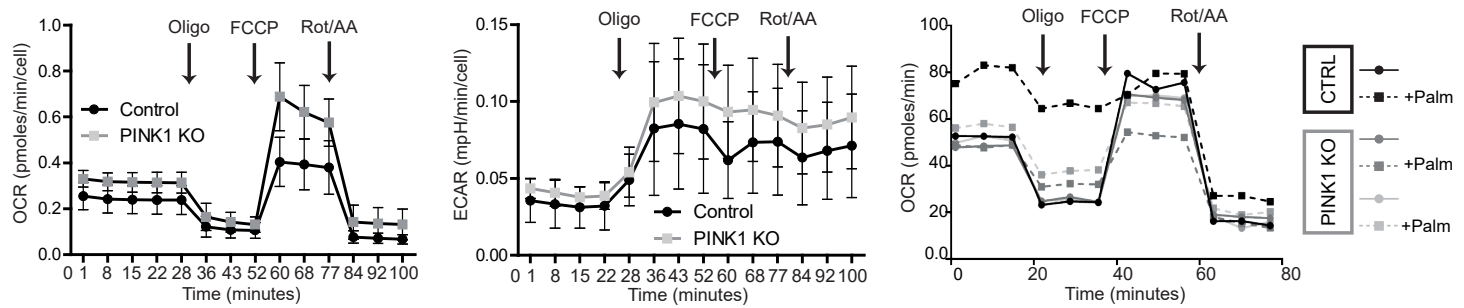

$\mathrm{B}$
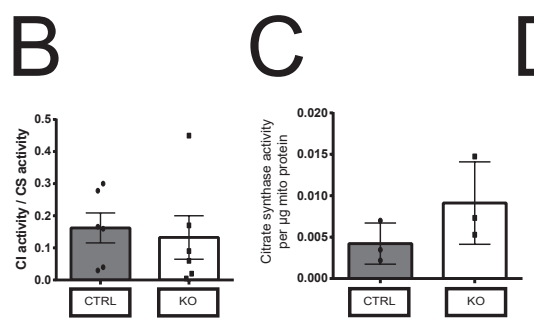

$\mathrm{D}$
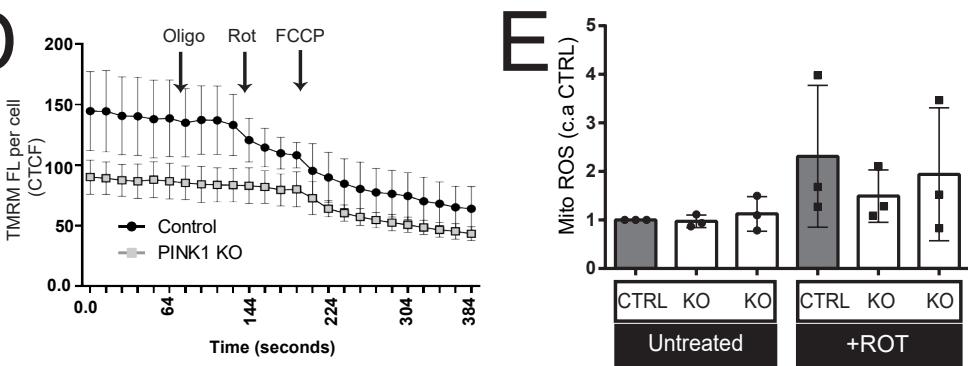

$\mathrm{F}$

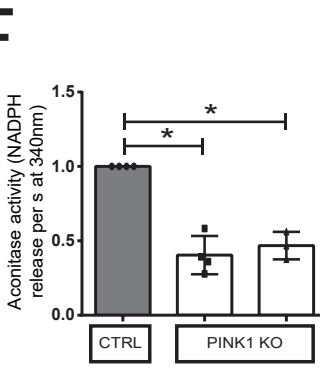

G

$\mathrm{H}$
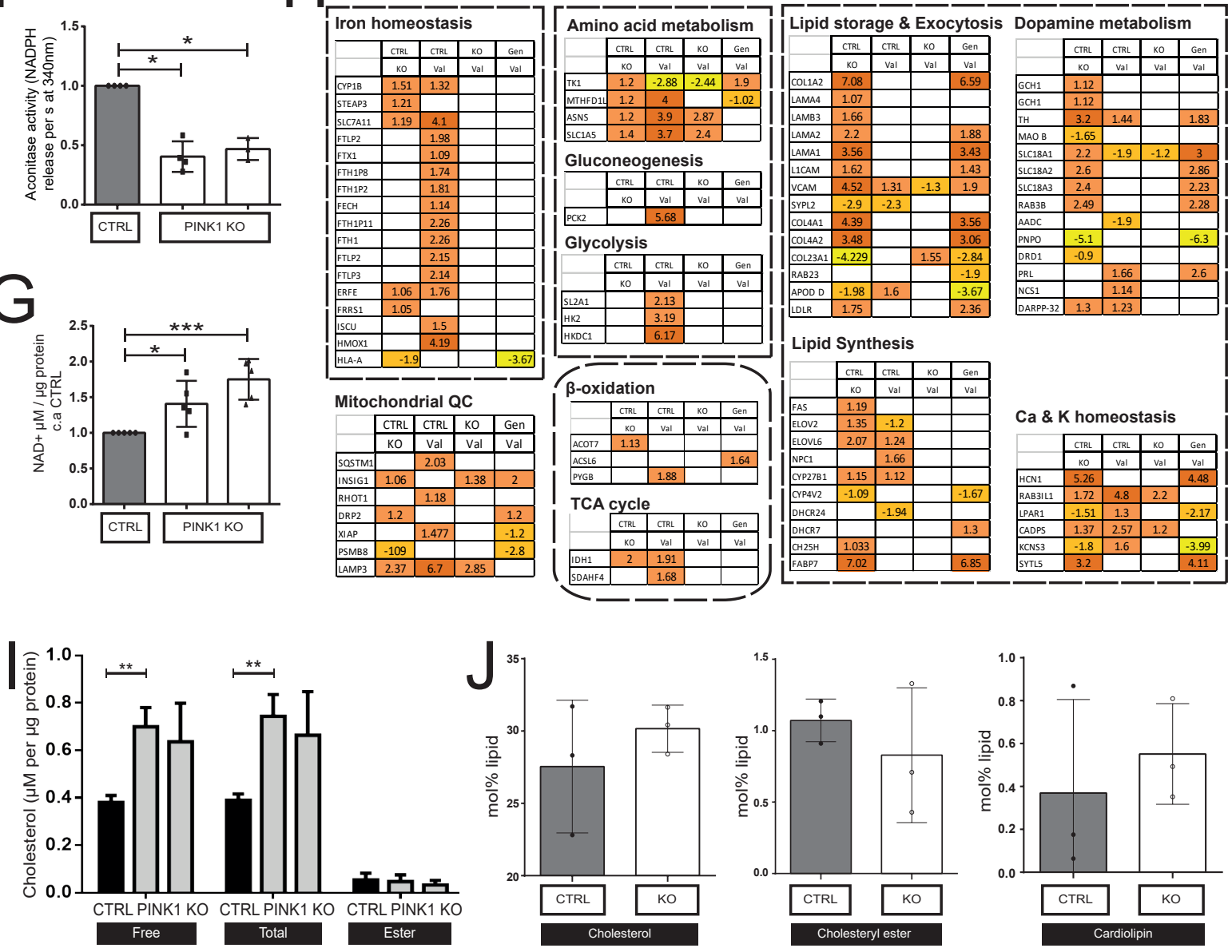

$\mathrm{K}$
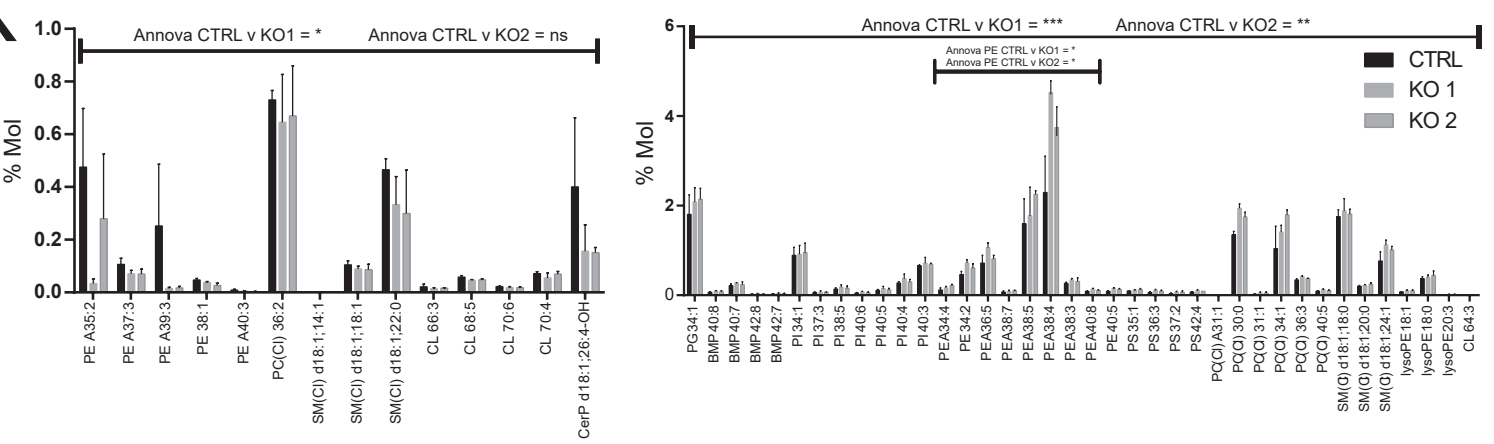
bioRxiv preprint a :anttps://doi.org/10.1101/814343; this version posted October 22, 2019. The copyright holder for this preprint (which

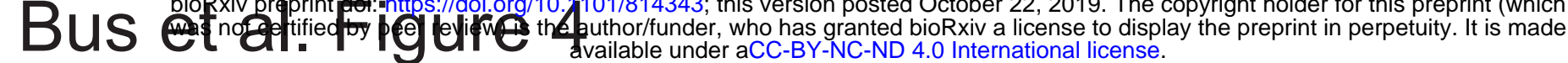

A
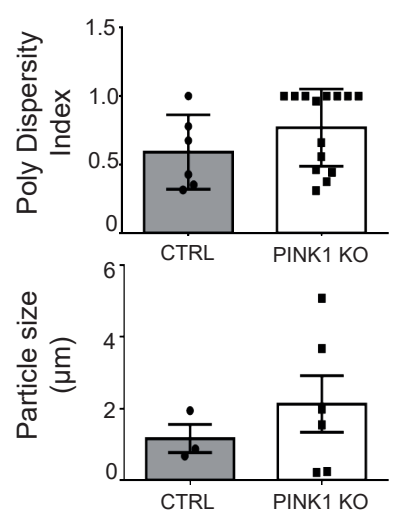

B Fraction

$\begin{array}{lllllllllllllll}1 & 2 & 3 & 4 & 5 & 6 & 7 & 8 & 9 & 10 & 11 & 12 & 13 & 14\end{array}$

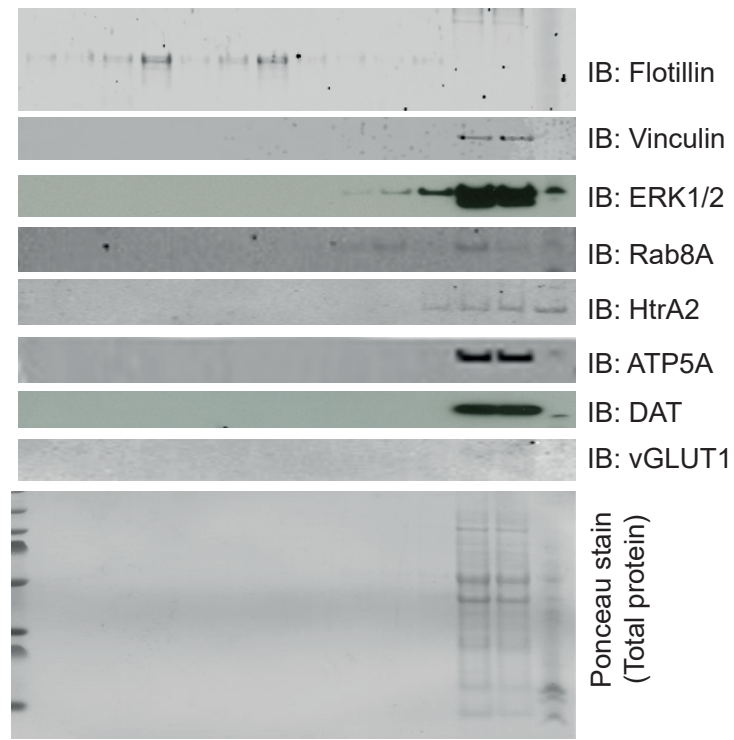

E + B-Cyclodextrin $1 \mathrm{mM} 16 \mathrm{~h}$

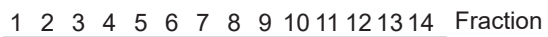

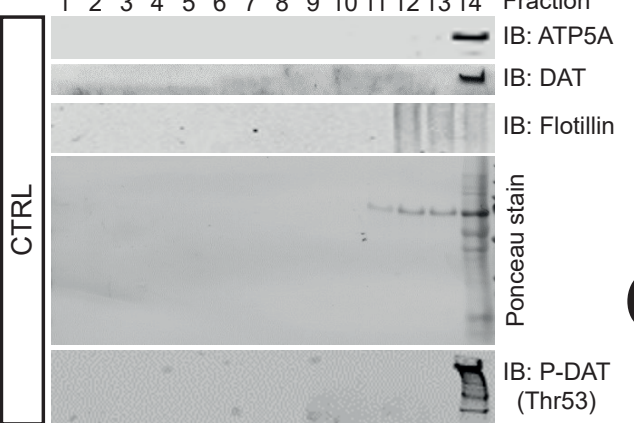

IB: P-DAT (Thr-53)

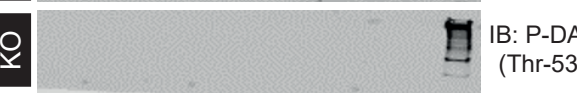

0
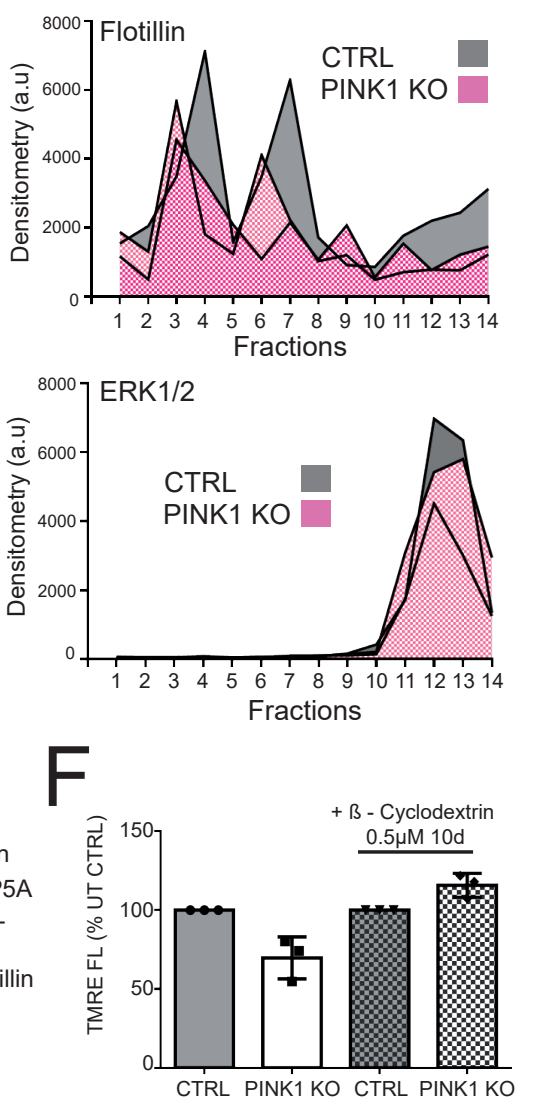

G

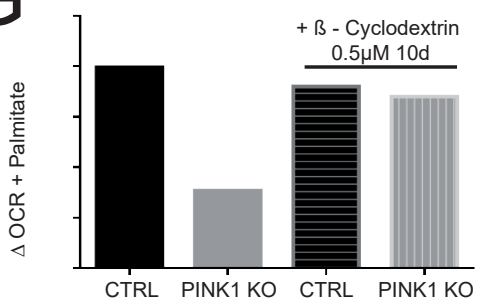

1
$\frac{y}{4}$

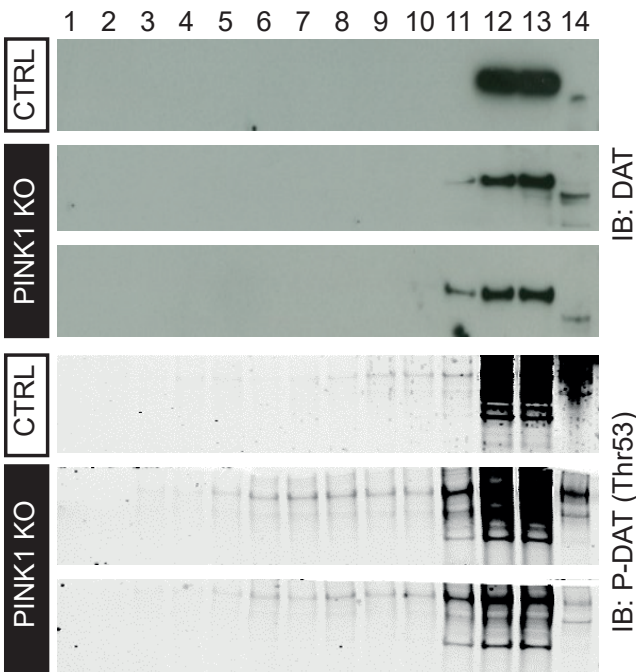




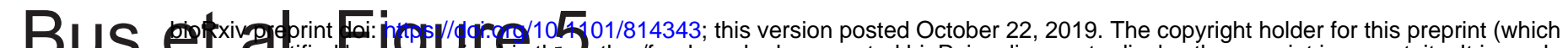

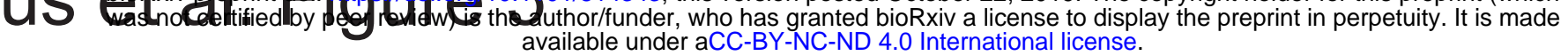

A

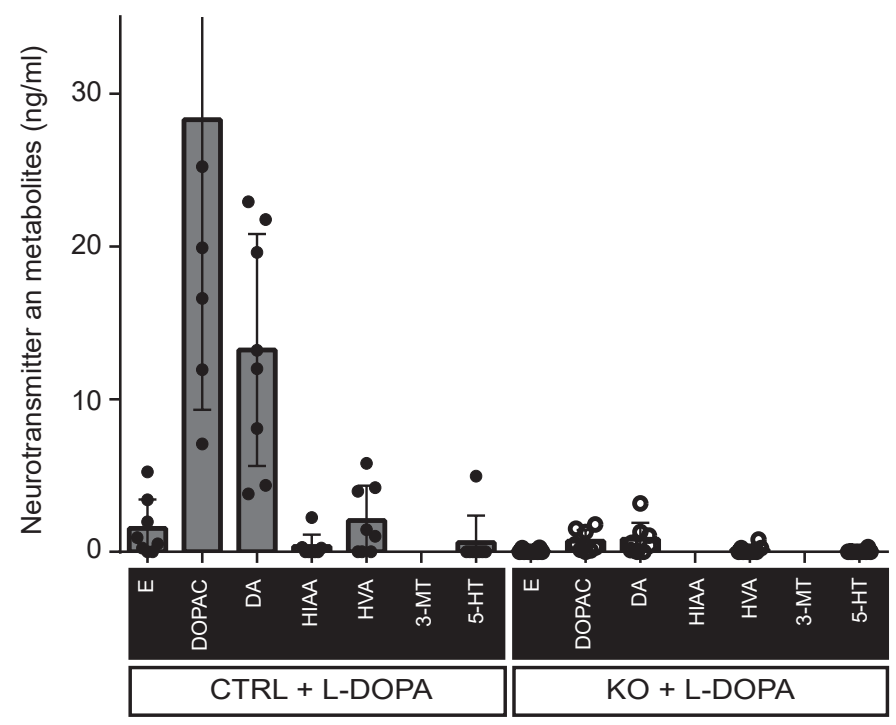

D

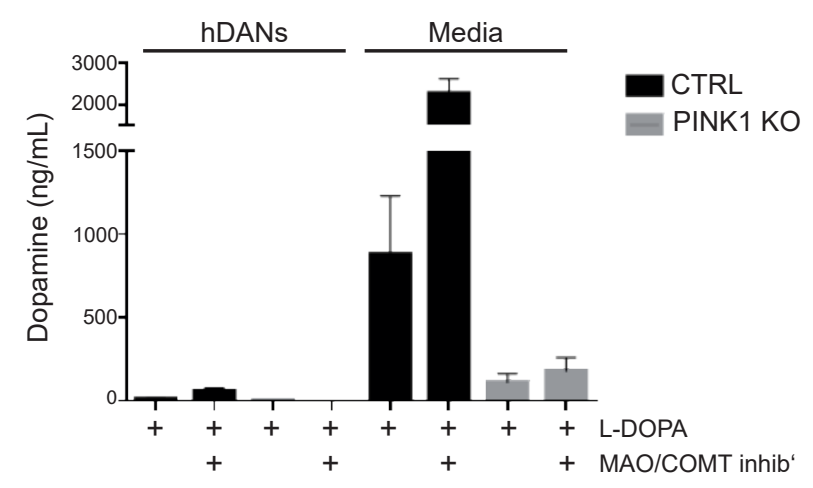

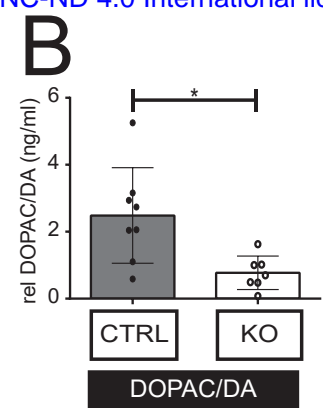
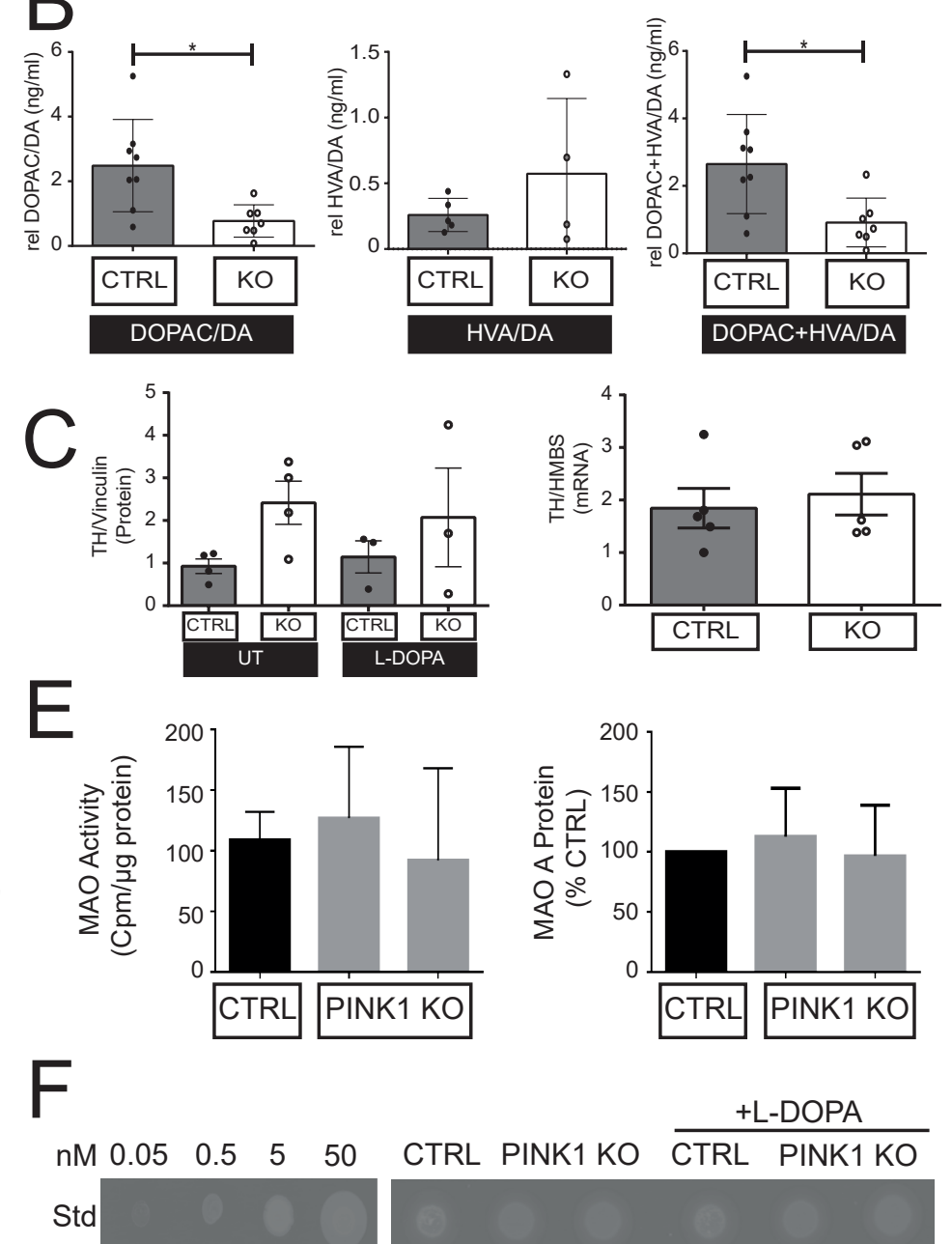

F
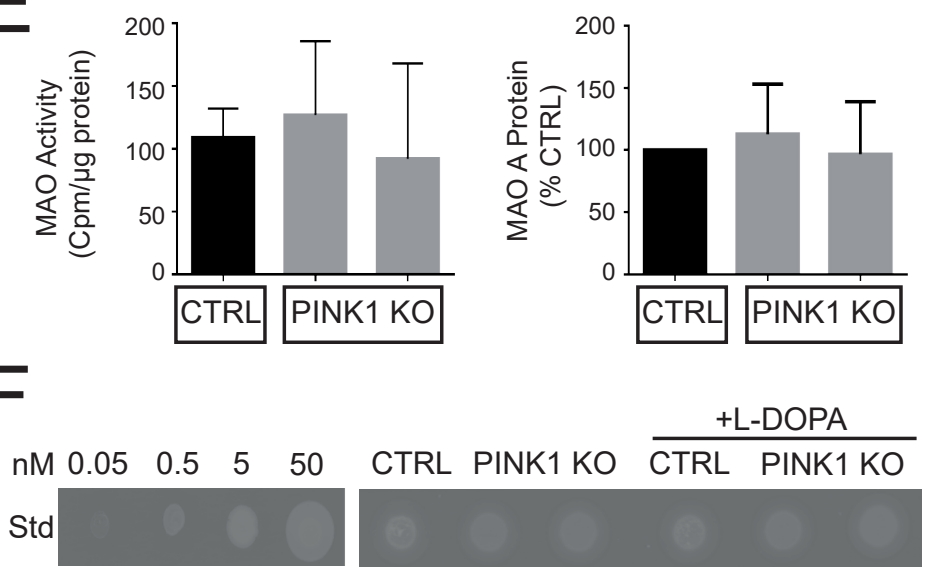

G

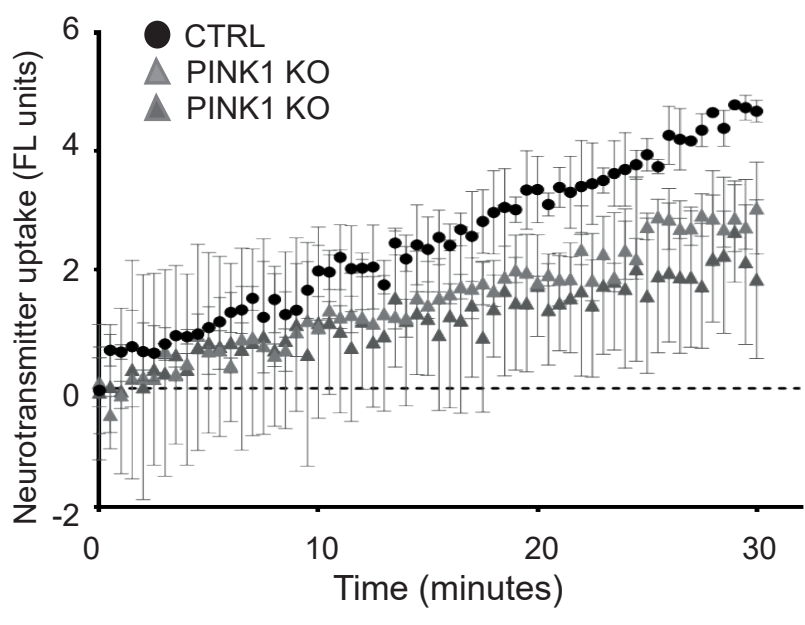

$\mathrm{H}$
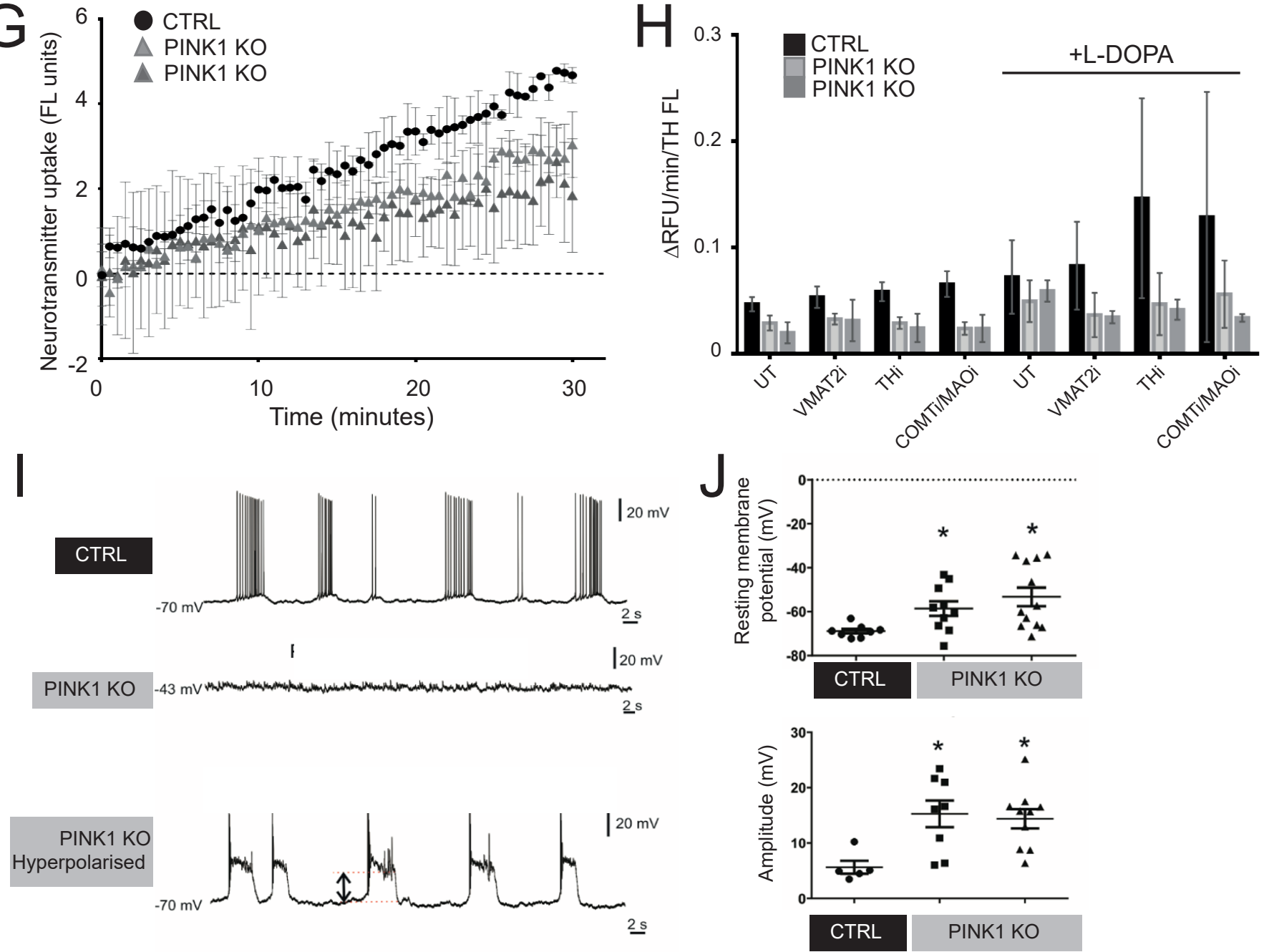